\title{
The Geodynamic Context of the Cenozoic Volcanism of the Cap-Vert Peninsula (Senegal)
}

\author{
Abdoulaye Ndiaye, Papa Malick Ngom \\ Department of Geology, Faculty of Sciences and Technology, Cheikh Anta Diop University, Dakar, Senegal \\ Email: ndiayeay69@yahoo.fr, papam.ngom@ucad.edu.sn
}

Received 9 September 2014; revised 4 October 2014; accepted 1 November 2014

Copyright (C) 2014 by authors and Scientific Research Publishing Inc.

This work is licensed under the Creative Commons Attribution International License (CC BY). http://creativecommons.org/licenses/by/4.0/

(c) (i) Open Access

\section{Abstract}

The Cenozoic alkaline volcanism of the Senegalo-Mauritania sedimentary basin presents an episodic long-lasting volcanic activity from the Eocene-Oligocene boundary up to the Quaternary. Two volcanic episodes are usually distinguished on stratigraphical grounds: a Miocene one and a Quaternary one separated by a period of quiescence of several million years corresponding to the main phase of lateritic weathering. The Tertiary lavas are highly silica-undersaturated alkaline rocks ranging from nephelinites to basanites. They contain nepheline and fassaite-type clinopyroxene in their mineralogy. The Quaternary lavas are more evolved with Hy-normative in comparison to the Tertiary ones; they are composed of basanites and medium to coarser-grained dolerites. The distribution of the REE and other incompatible elements are typical of alkaline lavas with generally strong LREE enrichment without significant Eu anomaly. The Cenozoic Cap-Vert lavas have OIB (oceanic islands basalts)-affinities as shown by their relatively radiogenic $\mathrm{Nd}$ and unradiogenic $\mathrm{Sr}$ characters closed to the HIMU-OIB. This suggests a HIMU-type end member in the magma sources which is fairly comparable to those erupted in the Canaries and Cape Verde archipelagoes during the same period.

\section{Keywords}

Cenozoic, Alkaline Volcanism, Oceanic Islands Basalts, Cap-Vert Peninsula, Senegalo-Mauritania Basin

\section{Introduction}

The West African Atlantic margin is spotted by three main alkaline volcanic provinces: the Canary Archipelago,

How to cite this paper: Ndiaye, A. and Ngom, P.M. (2014) The Geodynamic Context of the Cenozoic Volcanism of the Cap-Vert Peninsula (Senegal). International Journal of Geosciences, 5, 1521-1539. 
the Cape Verde Archipelago and the Cap-Vert peninsula (Dakar, Senegal). The Cap-Vert peninsula is located at the westernmost part of the Senegalo-Mauritania basin about $1000 \mathrm{~km}$ at the east of the Cape Verde Archipelago. The volcanic province (Figure 1) which extends further east onshore, is marked by large positive gravity anomalies in the Cap-Vert peninsula (110 mgal) and the Cayar Dome (160 mgal) identified by geophysics offshore [1]. It is subsequent to the opening of the Central Atlantic Ocean during the Lias, and related to the sub-meridional faults and the occurrence of E-W significant transverse fractures corresponding to the extension of oceanic transform faults on the continent.

Reference [2] was the first to suggest two eruptive rock series: one considered of Upper Cretaceous and the other of post-Meso-Nummulitic age. [3] confirmed the existence of these two series, but suggested stratigraphical

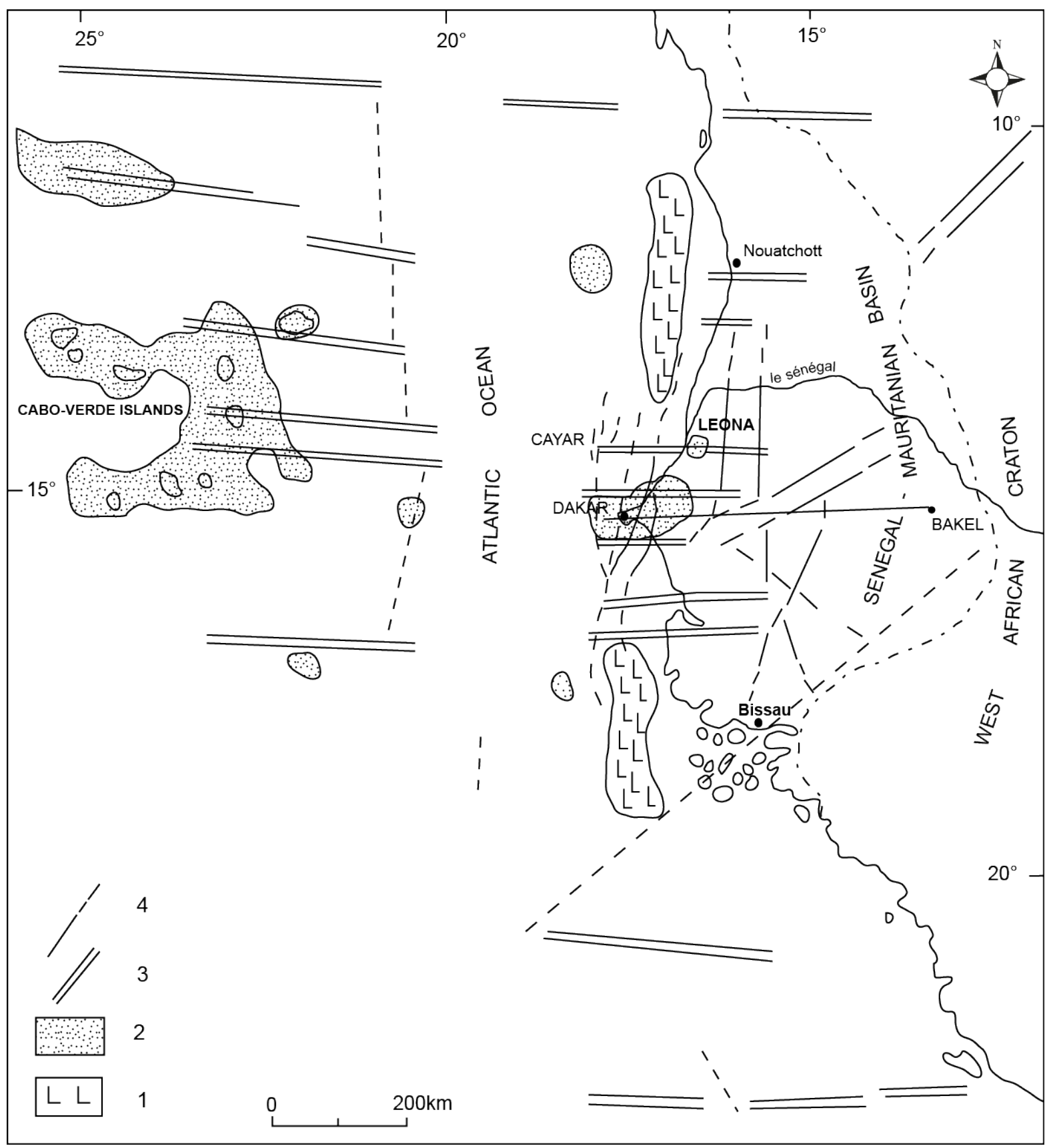

Figure 1. Coastal regions of Senegal: 1: zones of evaporates diapiric structures; 2: zones of regional positive gravimetric anomalies (>40 mgal); 3: Transform faults; 4: faults [4]. 
grounds during the Miocene age for the first occurence and an early Quaternary age for the second. Since the 50's, many descriptive studies of volcanic occurrences have been carried out [4].

References [4] [5] described an alkali syenite intrusion detectable only by drillingat Leona village (see Figure 1). This event can be connected with other activities occurring during the late Cretaceous along the West African Atlantic margin.

Reference [6] distinguished a Tertiary and a Quaternary volcanism separated by a ferruginous lateritic crust of Pliocene age. They presented an improved chronological framework for this volcanism based on K-Ar age determinations. These ages indicate an episodic long-lasting volcanic activity from Eocene-Oligocene boundary up to Quaternary in which the paroxysm is attained during the Serravallian and the Tortonian periods.

On the continental shelf, the activity of the Cenozoic volcanism is marked by intrusive rock sand hydrothermal vents in Paleozoic and Cretaceous sediments coinciding with the development of the Cayar Seamount and the volcanic eruptions on the Cape Verde archipelago [7] [8].

Several hypotheses have been advanced to explain the geodynamic significance of this volcanism. [9] [10] interpreted the Quaternary volcanism as anintra-plate volcanism associated with the migration of hot spots activities. This hypothesis is refuted by the absence of gular distribution of radiometric ages across the volcanic province [6]. They interpret the Cap-Vert peninsula volcanism as broadly similar to those of the Cape Verde and the Canary archipelagoes by their geodynamic setting age and magmatic characteristics.

This paper deals the interpretation from new geochemical data of the evolution of the Cenozoic volcanism of the Cap-Vert peninsula in relation with its geodynamic environment. The results are discussed and compared with the two other provinces of the Canary and Cape Verde Archipelagoes.

\section{Geological Setting}

The Senegalo-Mauritania sedimentary basin is geologically pretty well known [4] [6] [11]. Sedimentation is related to half-basin tectonic activity which began in the Middle Jurassic time and was active until recent time [12]. Gravimetric studies indicate the existence of a rapid thinning of the continental crust to the West [1]. The oceanic crust appears to open a hundred miles off Dakar area [13]. The basin (Figure 2) shows a monoclinal structure slightly dipping to the west and cut by sub-meridional normal faults, delimiting horsts and grabens and WNW-ESE faults corresponding to the extension of transform faults on the continent on which is localized the Cape Verde archipelago [11] [14]. These faults delimit the exonded block of the Cap-Vert peninsula that separates the Triassic-Liassic Casamance-Guinea evaporites basins whose deposition coincides with the birth of the rift stage of the Central Atlantic associated with an important phase of tholeiitic magmatic activity [15].

The magmatism acted along ancient zones of crustal weakness, which are related with inland extensions of transform faults. It is associated with two major tectonic events that occurred between the late Lutetian and late Eocene [16], and the Neogene [17]. Two major structures of NNE-SSW and NW-SE directions associated with other secondary NE-SW and NS in the Cap-Vert peninsula are brought out by [18]. These structures are reactivated faults which are cut successively by those of NNE-SSW and NW-SE orientations. In fact, most of those structures arelistric faults reactivated during the Alpine tectonic phase until a recent time.

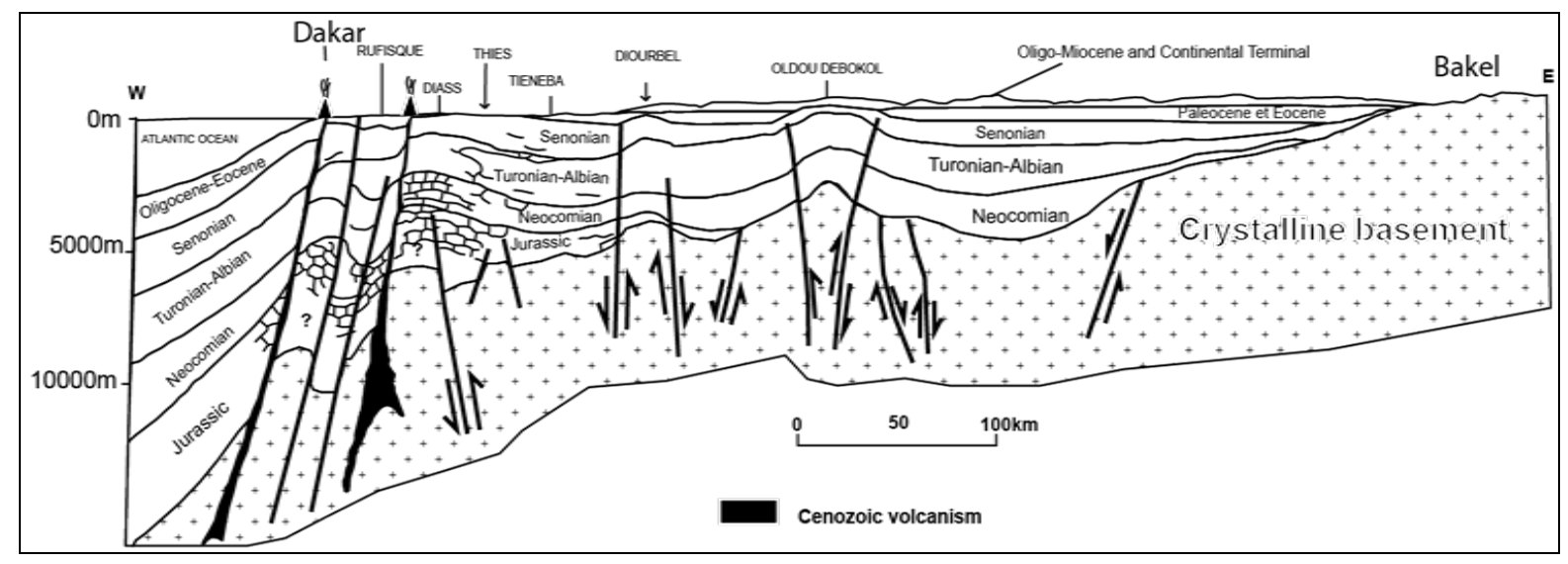

Figure 2. Schematic cross section of the Senegal basin from Bakel (E) to Dakar (W) ([12] modified). 


\section{Lithological and Petrographical Characteristics}

\subsection{The Tertiary Volcanism}

The Tertiary volcanism consists about thirty small occurrences of hectometric to kilometric extension scattered over an area about $100 \mathrm{~km}$ long from Dakar to the east of Thiès (Figure 3(a)). Four geographical groups are characterized by their different products:

1) Ankaratrite, nephelinite, basanitelavas flows or sills with columnar structure well spread in the Cap-Vert peninsula (Gorée, Cap Manuel, Iles des Madeleines, Pointe de Fann). The columns are generally vertical and regular shaped with polygonal section and some are bowed toward the edge of the flow;

2) basanite and nephelinitedykes with pegmatitoid veins and tuffs of limited extension in the Rufisque area (Cap des Biches, Diokoul); The dykes oriented $\mathrm{N}_{2} 0^{\circ}$, intrude the paleogene limestone sand the contact is marked by a weak thermal metamorphism. The rocks contain numerous vesicles filled of calcite and zeolite elongate NNE to NE trends.

3) Intrusions of basanites and tuffs located at the intersection of WNW-ESE and sub-meridian main fractures in the Ndiass horst area (Khazabe, Thiéo, Bandia);

4) Andin the Thiès region where the main outcrop would correspond to the remains of a lava lake located in a maar [19]. It is a N-S elongated cliff with a limited extension constituted of basanites, dolerite and pegmatitoidgabbros from the periphery to the inner sides of the pluton.

Petrographical features:

1) Nephelinites with aphyrictexture are usually associated with porphyritic (or pegmatitoids) nephelinites with coarser doleritic texture. In the microliticfacies, phenocrystalor microcrystalolivine ( $75 \%$ to $90 \%$ Fo) are usually corroded by anhyalinemesostasis. The clinopyroxene with fassaite to diopside compositions (Wo 47 - 55 En 30 38, Fs 9 - 17) represent the main mineral phase of the rock. An hedralnepheline is associated with needles of apatite and fine grains of olivine and clinopyroxene in the mesostasis. In the porphyritic facies, the clinopyroxenes of fassaitetype (Wo 50 - 55 En 29 - 38, Fs 11 - 16) exhibit occasionally hourglass twinning oroscillatoryzonation features. A few tabular minerals of plagioclase (An 50\% - 66\%) are associated with olivine (66\% to $84 \%$ Fo). Fine grains of apatite, zeolites, biotite and oxides are abundant in the rock.

2) The basanites are the most representative facies of this volcanism and are frequently associated with nephelinites. The common facies have aphyric texture and may have vacuolar structure with vesicles filled of calcite. The euhedral to subhedral crystals of olivine (67\% to $85 \%$ Fo) are sometimes surrounded by a thinrim ofidding site. The clinopyroxene with augite compositions (Wo 47 - 52, En 31 - 41, Fs 10 - 15) are corroded by

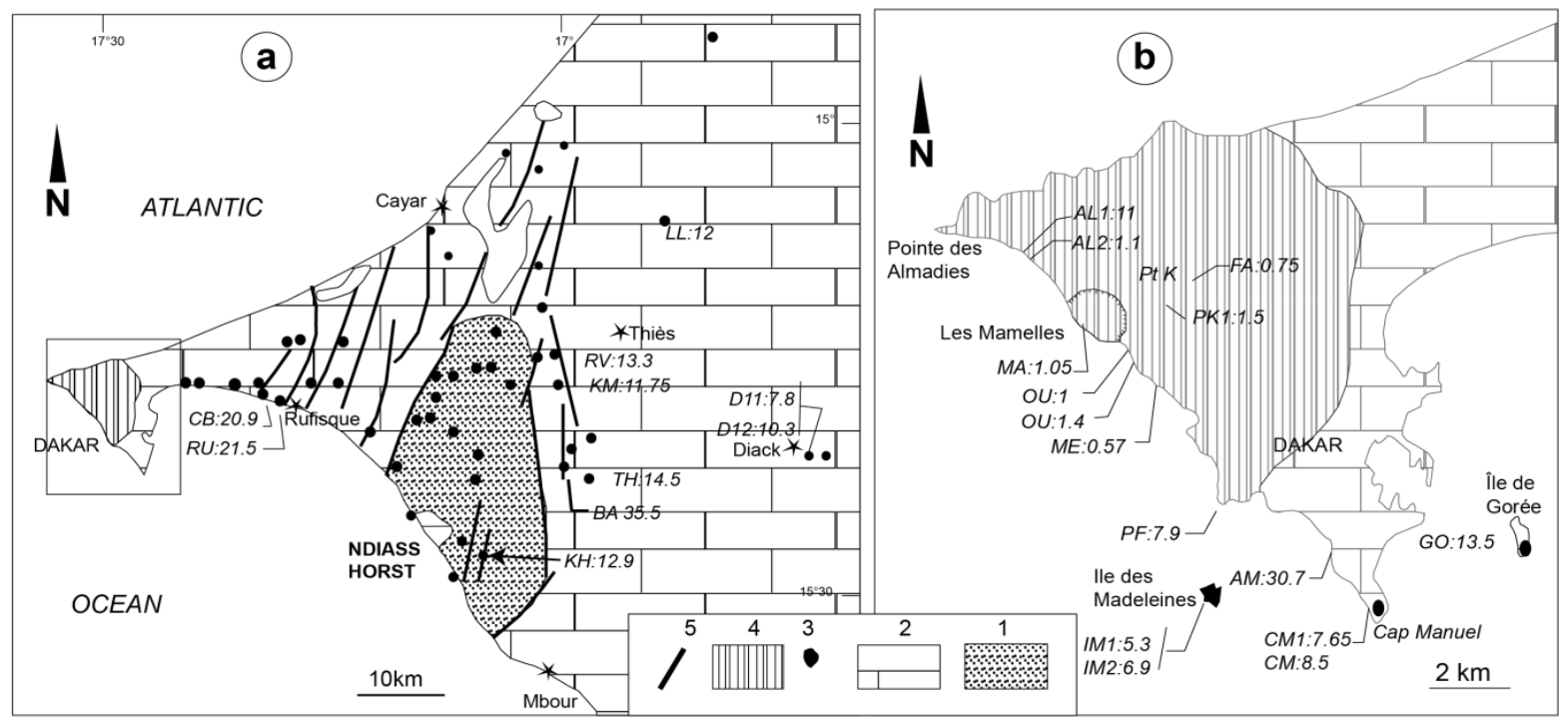

Figure 3. Outcrops of Cenozoic volcanic rocks in Cap-Vert peninsula [6]: (a) Cap-Vert peninsula and Thiès areas; (b) The Cenozoic volcanism of the Head of the Cap-Vert peninsula. 1: Mesozoic province (Ndiass Horst), 2: Tertiary carbonates, 3: Outcrops of Tertiary volcanic rocks dated (RU 21.5, K-Ar ages in Ma) or not dated, 4: Quaternary volcanic province with different K-Ar ages, 5: Faults. 
the mesostasis. The microlitic plagioclases (An 52\% - 80\%) are relatively abundant. Oxides are grouped into fine granulesorin inclusion in olivine or clinopyroxen eminerals. The hyaline ground mass contains microcrystals of olivine, clinopyroxene, plagioclase andoxides.

3) In the Diack pluton, the basanites, dolerites, and gabbrosare arranged into aconcentric structure from the periphery to the inner parts of the pluton. In the dolerites, the clinopyroxenes with augitic composition (Wo 44 - 45, En 40 - 44, Fs 10 - 14) and the olivine (60\% to $61 \%$ Fo) are grouped into the spaces between the plagioclase laths (An 44\% - 63\%). Oxides are abundant and exhibit a graphitic structure. The gabbros are composed of euhedral olivine (67\% Fo) locally surrounded by a fringe of alteration of idding site. The plagioclases (An $44 \%$ $52 \%$ ) are abundant and partially saussuritized. Abundant oxides are associated with clinopyroxene.

\subsection{The Quaternary Volcanism}

The Quaternary volcanism is confined at the Head of the Cap-Vert peninsula commonly named Mamelles volcano (Figure 3(b)). The flows are inter bedded with sand dunes of Quaternary age and laid on the end-Tertiary laterite [4] [20] [21].

From the bottom to the top, the Quaternary volcanismis divided into:

- A lower unit consisting of tuffs and thin flows of olivinebasalts known only by drilling;

- An intermediary unit is composed of dolerite sand basalts with aferruginous fringe of alteration;

- An upper unit composed of volcanic flows corresponding to the Mamelles volcano with several phases of volcanic activities: a) maar deposits established during an initial phase of phreatomagmatic eruption; b) strombolian activity building acinder cone with emission of lavas at the base of the cone; c) edification of alava lakein a large crater by a ring collapsein the cone; d) eruption of doleritic cone-sheet feeding the terminal effusive phase.

These products are structured into four columnar or lamellar flows separated byscories and ferruginized sandy-claymaterial [22]. They contain centimetric sized vesicles elongatedin the direction of the flow and filled of zeoliteorcalcite material. Nearby the volcanic cone, the basanites flows contain abundant xenoliths of peridotite and gabbro. The basanites are overlaid by two doleritic flows separated by a horizon of alteration consisting off ragments of scories, basanites and dolerites cemented by ferruginous clay material. The upper doleritehasa coarser structure with abundant vesicles exhibiting trails of degassing.

Petrographical features:

The basanites with fluidal texture are composed of olivine (64\% to $91 \%$ Fo) sometimes surrounded by a rim of iddingsite. Zoning clinopyroxene of augitic composition (Wo 45 - 50 En 36 - 44 Fs 10 - 15) are associated with abundant plagioclase (An 46\% - 76\%) in the upper flows. Oxides are usually included in plagioclase or in clinopyroxene crystals or dispersed in the mesostasis.

The dolerites with sub-ophitic to fluidal texture are composed of abundant laths of plagioclase with rare clinopyroxene and olivine crystals. In the upper dolerites, the plagioclase (An 27\% - 56\%) may represent the only mineral phase where it is associated with augitic clinopyroxene (Wo 31 - 47 En 38 - 49 Fs 11 - 24), olivine (58\% - 83\% Fo) partially idding sitized and Fe-Ti oxides.

\section{Geochemical and Isotopic Study}

\subsection{Analytical Methods}

Twenty three samples (present study) associated with eighteen samples ([6], unpublished data) were selected for major and trace element analysis (Table 1). Major and trace elements (including REE) were determined by ICP-AES and ICP-MS in CRPG (Nancy, France), following the procedure described by [23]. Nine representative samples were analyzed for $\mathrm{Nd}$ and $\mathrm{Sr}$ isotopic ratios ([6], unpublished data) in Clermont-Ferrand on a Micromass VG54E mass spectrometer using triple Ta-Re and single Ta filaments, respectively (Table 2). The entire process is described by [24].

\subsection{Major Elements}

The Cenozoic volcanic rocks exhibit distinctive geochemical compositions. The Tertiary lavas aresilica-undersaturated $(40 \%-48 \%)$ with $\mathrm{Na}_{2} \mathrm{O} / \mathrm{K}_{2} \mathrm{O}$ ratios values varying between 2.12 and 4.94 except in the porphyritic (or pegmatitoids) nephelinites where these values are more important (7.91 and 40.75). They are characterized by 
Table 1. Major and trace elements concentrations (DK samples are from Crévola et al., 1994, unpublished data).

\begin{tabular}{|c|c|c|c|c|c|c|c|c|c|c|c|c|c|c|c|c|c|c|c|c|c|c|c|}
\hline \multicolumn{24}{|c|}{ Tertiary volcanism } \\
\hline Samples. & 1 & 30 & 31 & 32 & 33 & 34 & 35 & 36 & 37 & 38 & 39 & DK18 & DK13 & DK15 & DK14 & DK1 & DK17 & DK16 & DK3 & DK2 & DK6 & DK7 & DK8 \\
\hline $\mathrm{SiO}_{2}$ & 41.72 & 41.58 & 39.26 & 41.14 & 42.46 & 39.39 & 39.87 & 38.76 & 42.33 & 47.85 & 47.22 & 40.46 & 41.50 & 39.80 & 43.34 & 39.96 & 45.31 & 46.91 & 40.81 & 40.62 & 40.60 & 39.56 & 47.21 \\
\hline $\mathrm{Al}_{2} \mathrm{O}_{3}$ & 11.51 & 11.71 & 13.12 & 11.55 & 13.49 & 12.14 & 12.55 & 15.91 & 12.23 & 13.62 & 15.95 & 11.67 & 12.00 & 12.80 & 14.02 & 12.63 & 13.20 & 13.20 & 12.03 & 12.96 & 17.27 & 11.26 & 13.55 \\
\hline $\mathrm{Fe}_{2} \mathrm{O}_{3}$ & 13.41 & 12.56 & 10.42 & 12.39 & 11.41 & 11.57 & 11.91 & 12.09 & 12.37 & 11.57 & 11.93 & 11.67 & 13.10 & 11.80 & 12.03 & 11.85 & 11.43 & 11.14 & 10.94 & 11.49 & 10.39 & 11.96 & 11.85 \\
\hline $\mathrm{MnO}$ & 0.19 & 0.19 & 0.17 & 0.18 & 0.18 & 0.19 & 0.20 & 0.22 & 0.20 & 0.16 & 0.19 & 0.19 & 0.20 & 2.00 & 0.19 & 0.17 & 0.17 & 0.16 & 0.17 & 0.17 & 0.17 & 0.20 & 0.16 \\
\hline $\mathrm{MgO}$ & 12.72 & 12.73 & 7.69 & 12.25 & 9.15 & 11.71 & 10.62 & 5.80 & 10.77 & 9.80 & 3.52 & 12.07 & 11.30 & 11.10 & 9.26 & 11.05 & 11.94 & 11.23 & 12.82 & 11.14 & 4.90 & 13.53 & 9.69 \\
\hline $\mathrm{CaO}$ & 12.84 & 12.33 & 15.95 & 12.56 & 12.05 & 14.97 & 14.55 & 13.77 & 12.32 & 10.06 & 8.74 & 15.38 & 12.40 & 14.40 & 12.72 & 14.83 & 11.64 & 11.99 & 15.53 & 14.86 & 11.08 & 14.33 & 10.48 \\
\hline $\mathrm{Na}_{2} \mathrm{O}$ & 2.64 & 2.73 & 3.30 & 2.72 & 3.46 & 1.97 & 2.94 & 4.69 & 4.10 & 2.86 & 4.86 & 2.84 & 3.54 & 2.83 & 3.39 & 3.41 & 2.83 & 2.85 & 3.12 & 3.29 & 5.58 & 3.04 & 3.16 \\
\hline $\mathrm{K}_{2} \mathrm{O}$ & 0.96 & 0.85 & 1.56 & 0.69 & 1.00 & 0.25 & 0.26 & 0.12 & 0.88 & 0.76 & 2.02 & 1.08 & 0.92 & 0.27 & 1.17 & 7.00 & 1.14 & 0.90 & 0.76 & 0.68 & 2.50 & 0.73 & 0.64 \\
\hline $\mathrm{TiO}_{2}$ & 2.50 & 1.82 & 2.07 & 1.80 & 1.96 & 1.96 & 2.05 & 1.99 & 2.22 & 1.55 & 2.49 & 2.13 & 2.50 & 2.04 & 2.04 & 2.37 & 2.14 & 1.80 & 1.97 & 3.08 & 1.68 & 1.93 & 1.43 \\
\hline $\mathrm{P}_{2} \mathrm{O}_{5}$ & 0.83 & 0.90 & 2.77 & 0.85 & 0.84 & 0.89 & 1.00 & 1.59 & 0.58 & 0.28 & 1.02 & 1.05 & 0.90 & 1.05 & 0.91 & 0.94 & 0.58 & 0.57 & 0.93 & 0.76 & 1.62 & 1.04 & 0.51 \\
\hline $\mathrm{PF}$ & 0.88 & 1.77 & 2.61 & 2.80 & 2.92 & 4.31 & 4.10 & 5.17 & 2.37 & 1.26 & 1.87 & 1.84 & 1.66 & 4.42 & 2.66 & 1.59 & 0.66 & 0.76 & 1.32 & 1.42 & 3.61 & 2.18 & 1.03 \\
\hline Total & 100 & 99.16 & 98.92 & 98.93 & 98.91 & 99.37 & 100 & 100 & 100 & 99.76 & 99.80 & 100 & 100 & 100.71 & 101.73 & 99.48 & 101.04 & 101.51 & 100 & 100 & 99.40 & 99.76 & 99.71 \\
\hline $\mathrm{NaO} / \mathrm{K}_{2} \mathrm{O}$ & 2.75 & 3.19 & 2.12 & 3.96 & 3.46 & 7.91 & 11.12 & 40.75 & 4.65 & 3.78 & 2.41 & 2.63 & 3.85 & 10.48 & 2.90 & 5.01 & 2.48 & 3.17 & 4.11 & 4.84 & 2.23 & 4.16 & 4.94 \\
\hline $\mathrm{Ne}^{\prime}$ & 11.7 & 10.5 & 13.7 & 9.75 & 10.1 & 8.63 & 13.4 & 21.2 & 19.2 & 0 & 10.6 & 13 & 15.3 & 13 & 12.5 & 15.6 & 7.11 & 4.43 & 14.3 & 15.1 & 25.58 & 1.35 & 13.9 \\
\hline Co & 83.3 & 77.9 & 82.6 & 76.6 & 73.8 & 69.8 & 68.2 & 55.9 & 78.4 & 123 & 49.9 & 72 & 76 & 73 & 60 & 82 & 69 & 67 & 74 & 65 & 42 & 64 & 59 \\
\hline $\mathrm{Cr}$ & 447 & 399 & 99.6 & 386 & 217 & 400 & 362 & 12.2 & 393 & 467 & 43 & 386 & 358 & 335 & 203 & 258 & 577 & 548 & 577 & 531 & 18 & 356 & 49 \\
\hline Cs & 0.37 & 0.37 & 0.26 & 0.36 & 0.93 & 0.39 & 0.27 & 0.48 & 1.26 & 0.88 & 1.53 & nd & nd & nd & nd & nd & nd & nd & nd & nd & nd & nd & nd \\
\hline $\mathrm{Hf}$ & 5.28 & 3.30 & 3.75 & 3.62 & 4.55 & 6.69 & 4.53 & 3.05 & 4.69 & 2.22 & 4.67 & nd & nd & nd & nd & nd & nd & nd & nd & nd & nd & nd & nd \\
\hline $\mathrm{Nb}$ & 80.9 & 61.1 & 108 & 61.6 & 99.8 & 118 & 156 & 236 & 122 & 39.1 & 156 & 91 & 119 & 118 & 104 & 96 & 88 & 54 & 103 & 98 & 134 & 46 & 84 \\
\hline $\mathrm{Ni}$ & 371 & 301 & 105 & 289 & 142 & 217 & 199 & 54.4 & 260 & 305 & 50.5 & 298 & 293 & 172 & 120 & 210 & 307 & 306 & 269 & 201 & 59 & 236 & 308 \\
\hline $\mathrm{Rb}$ & 27.9 & 23.1 & 55.6 & 17.6 & 26.7 & 14.2 & 12.1 & 8.82 & 44.4 & 24.9 & 63.9 & 31 & 30 & 11 & 30 & 23 & 34 & 18 & 23 & 27 & 89 & 24 & 29 \\
\hline $\mathrm{Sr}$ & 802 & 828 & 1540 & 872 & 1997 & 2732 & 2799 & 3571 & 802 & 552 & 843 & 1583 & 709 & 2812 & 1490 & 1302 & 784 & 694 & 1073 & 1011 & 1774 & 579 & 1088 \\
\hline Ta & 7.26 & 4.14 & 7.45 & 4.61 & 6.11 & 6.81 & 7.74 & 11.01 & 9.88 & 3.39 & 11.38 & nd & nd & nd & nd & nd & nd & nd & nd & nd & nd & nd & nd \\
\hline Th & 5.27 & 5.11 & 9.67 & 5.44 & 11.85 & 10.77 & 12.17 & 18.23 & 5.74 & 2.20 & 8.80 & nd & nd & nd & nd & nd & nd & nd & nd & nd & nd & nd & nd \\
\hline $\mathrm{U}$ & 1.25 & 1.24 & 2.09 & 1.28 & 3.64 & 2.59 & 3.18 & 4.79 & 1.38 & 0.53 & 2.10 & nd & nd & nd & nd & nd & nd & nd & nd & nd & nd & nd & nd \\
\hline $\mathrm{V}$ & 228 & 191 & 225 & 180 & 196 & 227 & 268 & 314 & 249 & 218 & 232 & 233 & 220 & 242 & 206 & 219 & 242 & 220 & 220 & 284 & 202 & 179 & 197 \\
\hline $\mathrm{Y}$ & 25 & 25 & 38.6 & 25.9 & 28.8 & 29.7 & 33.6 & 40.4 & 24.9 & 20.6 & 36.4 & 29 & 27 & 31 & 31 & 27 & 24 & 23 & 25 & 22 & 33 & 29 & 21 \\
\hline $\mathrm{Zr}$ & 212 & 152 & 201 & 155 & 222 & 329 & 257 & 257 & 227 & 99.2 & 250 & 182 & 228 & 226 & 212 & 165 & 231 & 156 & 200 & 212 & 156 & 89 & 178 \\
\hline $\mathrm{La}$ & 45.62 & 55.40 & 112.90 & 57.99 & 103 & 87.63 & 97.77 & 140 & 42.32 & 17.59 & 65.04 & 80.90 & 49.50 & 88.30 & 101.00 & 83.52 & 47.20 & 38.40 & 82.41 & 84.64 & 107 & 44.34 & 74.00 \\
\hline Ce & 88.57 & 107.70 & 216.10 & 113.40 & 174 & 164 & 181.60 & 252 & 78.71 & 34.32 & 118.30 & 158 & 95.2 & 167 & 176 & 155 & 92.6 & 77.5 & 152 & 101 & 184.8 & 74.65 & 141 \\
\hline $\operatorname{Pr}$ & 10.43 & 12.40 & 23.85 & 13.05 & 18.60 & 18.27 & 19.71 & 26.42 & 8.90 & 4.08 & 12.90 & nd & nd & nd & nd & nd & nd & nd & nd & nd & nd & nd & nd \\
\hline $\mathrm{Nd}$ & 41.61 & 48.29 & 89.79 & 50.68 & 67.47 & 67.26 & 72.25 & 92.71 & 34.46 & 16.79 & 48.19 & 66.10 & 41.60 & 67.90 & 67.30 & 63.27 & 40.17 & 34.30 & 63.12 & 46.02 & 72.14 & 28.67 & 61.60 \\
\hline $\mathrm{Sm}$ & 8.44 & 9.02 & 15.65 & 9.54 & 11.42 & 11.45 & 11.92 & 14.58 & 6.82 & 3.78 & 9.08 & 9.23 & 7.75 & 9.80 & 10.70 & 12.25 & 5.17 & 5.98 & 11.75 & 9.43 & 13.02 & 6.20 & 11.93 \\
\hline $\mathrm{Eu}$ & 2.73 & 2.79 & 4.68 & 2.90 & 3.52 & 3.42 & 3.65 & 4.26 & 2.28 & 1.37 & 2.98 & 2.47 & 2.15 & 2.52 & 2.73 & 3.51 & 1.40 & 1.78 & 3.26 & 2.77 & 3.72 & 1.88 & 3.52 \\
\hline Gd & 7.42 & 7.46 & 12.55 & 7.57 & 9.14 & 9.00 & 9.60 & 11.60 & 6.20 & 4.02 & 8.47 & 7.90 & 6.81 & 8.08 & 8.48 & 9.77 & 4.90 & 5.68 & 9.18 & 8.01 & 10.07 & 5.73 & 9.59 \\
\hline $\mathrm{Tb}$ & 1.02 & 1.02 & 1.62 & 1.08 & 1.18 & 1.22 & 1.28 & 1.52 & 0.87 & 0.62 & 1.21 & $\mathrm{n}$ & $\mathrm{n}$ & $\mathrm{n}$ & $n$ & $n$ & nd & nd & nd & nd & nd & nd & nd \\
\hline Dy & 5.39 & 5.34 & 8.38 & 5.64 & 6.13 & 6.21 & 6.55 & 7.77 & 4.73 & 3.58 & 6.78 & 5.68 & 4.95 & 6.07 & 5.87 & 5.68 & 4.46 & 4.21 & 5.38 & 4.92 & 6.71 & 4.23 & 6.10 \\
\hline Ho & 0.92 & 0.91 & 1.37 & 0.95 & 1.02 & 1.05 & 1.11 & 1.33 & 0.82 & 0.67 & 1.20 & nd & nd & nd & nd & nd & nd & $n$ & nd & nd & nd & nd & nd \\
\hline Er & 2.32 & 2.21 & 3.36 & 2.37 & 2.62 & 2.67 & 2.83 & 3.49 & 2.05 & 1.76 & 3.11 & 2.32 & 2.24 & 2.70 & 2.67 & 2.36 & 1.85 & 1.88 & 2.24 & 2.00 & 2.94 & 1.94 & 2.49 \\
\hline $\mathrm{Tm}$ & 0.30 & 0.29 & 0.43 & 0.31 & 0.35 & 0.37 & 0.38 & 0.47 & 0.28 & 0.24 & 0.42 & $\mathrm{n}$ & $\mathrm{n}$ & nd & nd & nd & nd & nd & nd & nd & nd & nd & nd \\
\hline $\mathrm{Yb}$ & 1.83 & 1.79 & 2.51 & 1.87 & 2.10 & 2.26 & 2.36 & 2.92 & 1.70 & 1.46 & 2.66 & 1.34 & 1.71 & 1.89 & 1.99 & 1.72 & 1.14 & 1.35 & 1.64 & 1.44 & 2.23 & 1.54 & 1.81 \\
\hline $\mathrm{Lu}$ & 0.28 & 0.26 & 0.35 & 0.28 & 0.31 & 0.34 & 0.34 & 0.42 & 0.25 & 0.22 & 0.38 & 0.16 & 0.24 & 0.24 & 0.27 & 0.31 & 0.14 & 0.19 & 0.30 & 0.25 & 0.37 & 0.26 & 0.29 \\
\hline
\end{tabular}


Table 1 (end) (DK samples are from [6] (unpublished data).

\begin{tabular}{|c|c|c|c|c|c|c|c|c|c|c|c|c|c|c|c|c|c|c|}
\hline \multicolumn{19}{|c|}{ Quaternary volcanism } \\
\hline Sample & 6 & 17 & 25 & 26 & 27 & 28 & 14 & $\mathbf{A}$ & 16 & 19 & 29 & 18 & DK12 & DK11 & DK5 & DK10 & DK4 & DK9 \\
\hline $\mathrm{SiO}_{2}$ & 48.23 & 47.54 & 46.91 & 47.90 & 48.24 & 47.97 & 50.27 & 49.35 & 51.30 & 50.65 & 50.51 & 47.13 & 48.42 & 51.72 & 46.90 & 49.84 & 48.20 & 51.75 \\
\hline $\mathrm{Al}_{2} \mathrm{O}_{3}$ & 14.80 & 15.04 & 14.60 & 14.87 & 14.69 & 14.66 & 17.27 & 14.90 & 14.72 & 14.69 & 15.44 & 14.82 & 15.18 & 15.21 & 14.92 & 15.60 & 15.08 & 14.66 \\
\hline $\mathrm{Fe}_{2} \mathrm{O}_{3}$ & 10.41 & 10.61 & 9.81 & 10.32 & 10.96 & 10.82 & 12.08 & 11.30 & 10.44 & 9.71 & 10.63 & 10.26 & 10.91 & 10.30 & 10.46 & 10.71 & 10.89 & 10.91 \\
\hline $\mathrm{MnO}$ & 0.13 & 0.14 & 0.13 & 0.13 & 0.14 & 0.14 & 0.11 & 0.15 & 0.14 & 0.10 & 0.12 & 0.14 & 0.12 & 0.12 & 0.12 & 0.13 & 0.13 & 0.13 \\
\hline MgO & 7.88 & 7.99 & 7.83 & 7.40 & 8.41 & 8.20 & 3.52 & 7.04 & 7.33 & 7.39 & 5.56 & 7.98 & 7.18 & 7.24 & 8.21 & 7.26 & 7.76 & 7.01 \\
\hline $\mathrm{CaO}$ & 7.33 & 8.00 & 9.42 & 8.75 & 8.59 & 7.91 & 6.83 & 8.88 & 9.01 & 8.50 & 9.07 & 9.07 & 7.84 & 8.96 & 8.32 & 9.36 & 8.36 & 8.73 \\
\hline $\mathrm{Na}_{2} \mathrm{O}$ & 4.06 & 4.79 & 4.30 & 3.89 & 3.66 & 4.51 & 3.78 & 3.72 & 3.94 & 3.78 & 4.05 & 4.16 & 5.05 & 4.01 & 4.20 & 3.84 & 4.74 & 3.95 \\
\hline $\mathrm{K} 2 \mathrm{O}$ & 1.97 & 1.46 & 1.52 & 1.43 & 1.24 & 1.68 & 0.59 & 1.04 & 0.69 & 0.76 & 0.47 & 1.45 & 1.62 & 0.61 & 1.43 & 0.70 & 1.51 & 0.63 \\
\hline $\mathrm{TiO}_{2}$ & 2.18 & 2.13 & 1.89 & 1.90 & 1.81 & 2.13 & 1.61 & 1.70 & 1.52 & 1.49 & 1.55 & 1.88 & 2.12 & 1.35 & 2.00 & 1.43 & 1.86 & 1.54 \\
\hline $\mathrm{P}_{2} \mathrm{O}_{5}$ & 0.69 & 0.60 & 0.56 & 0.59 & 0.50 & 0.55 & 0.30 & 0.47 & 0.33 & 0.34 & 0.33 & 0.58 & 0.53 & 0.36 & 0.60 & 0.34 & 0.61 & 0.40 \\
\hline $\mathrm{PF}$ & 2.66 & 1.07 & 1.84 & 1.97 & 1.19 & 0.88 & 3.63 & 1.16 & -0.04 & 1.86 & 1.85 & 1.69 & 0.58 & nd & 2.48 & 0.57 & 0.51 & 0.03 \\
\hline Total & 100 & 99.36 & 98.80 & 99.13 & 99.41 & 99.43 & 99.99 & 99.70 & 99.39 & 99.28 & 99.56 & 99.15 & 99.55 & 99.88 & 99.64 & 99.78 & 99.65 & 99.74 \\
\hline $\mathrm{NaO} / \mathrm{K}_{2} \mathrm{O}$ & 2.06 & 3.29 & 2.83 & 2.72 & 2.95 & 2.69 & 6.37 & 3.58 & 5.68 & 4.97 & 8.70 & 2.87 & 3.12 & 6.57 & 2.94 & 5.49 & 3.14 & 6.27 \\
\hline $\mathrm{Ne}^{\prime}$ & 2.93 & 8.01 & 8.33 & 2.62 & 1.29 & 8.82 & 0 & 0 & 0 & 0 & 0 & 6.52 & 8.52 & 0 & 5.72 & 0 & 7.76 & 0 \\
\hline Co & 54.9 & 56.9 & 68.3 & 84.9 & 68.8 & 67.5 & 54.7 & 57.1 & 70.5 & 47.2 & 60.6 & 50.8 & 55 & 63 & 51 & 65 & 60 & 60 \\
\hline $\mathrm{Cr}$ & 238 & 253 & 225 & 239 & 262 & 307 & 274 & 257 & 237 & 215 & 242 & 215 & 246 & 261 & 236 & 244 & 257 & 35 \\
\hline Cs & 0.66 & 1.66 & 0.36 & 0.43 & 0.20 & 0.58 & $<\mathrm{LD}$ & 0.23 & $<\mathrm{LD}$ & $<\mathrm{LD}$ & $<\mathrm{LD}$ & 0.41 & nd & nd & nd & nd & nd & nd \\
\hline $\mathrm{Hf}$ & 4.34 & 4.08 & 3.45 & 3.59 & 3.14 & 3.81 & 2.55 & 2.88 & 2.15 & 2.30 & 2.29 & 3.73 & nd & nd & nd & nd & nd & nd \\
\hline $\mathrm{Nb}$ & 66.5 & 58.2 & 52.8 & 53.3 & 46.1 & 55.8 & 30.1 & 41.2 & 30.6 & 31.1 & 32.1 & 51.5 & 56 & 31 & 59 & 27 & 57 & 33 \\
\hline $\mathrm{Ni}$ & 226 & 231 & 215 & 212 & 237 & 268 & 151 & 218 & 136 & 125 & 116 & 190 & 229 & 146 & 199 & 197 & 227 & 140 \\
\hline $\mathrm{Rb}$ & 57.4 & 17.2 & 40.9 & 38 & 28.2 & 45 & 12.3 & 25.4 & 15.3 & 16.9 & 4.47 & 37.4 & 39 & 17 & 35 & 19 & 44 & 17 \\
\hline $\mathrm{Sr}$ & 858 & 749 & 772 & 741 & 674 & 714 & 442 & 595 & 466 & 433 & 487 & 742 & 710 & 496 & 768 & 491 & 758 & 451 \\
\hline Ta & 5.01 & 4.54 & 3.84 & 3.92 & 3.20 & 4.19 & 1.76 & 2.71 & 1.89 & 1.80 & 1.86 & 3.80 & nd & nd & nd & nd & nd & nd \\
\hline Th & 4.56 & 5.23 & 4.07 & 4.17 & 3.51 & 3.79 & 2.88 & 2.87 & 2.77 & 3.18 & 3.16 & 4.60 & nd & nd & nd & nd & nd & nd \\
\hline $\mathrm{U}$ & 0.63 & 1.19 & 0.79 & 0.94 & 0.72 & 0.86 & 0.67 & 0.73 & 0.48 & 0.82 & 0.67 & 1.09 & nd & nd & nd & nd & nd & nd \\
\hline $\mathrm{V}$ & 114 & 141 & 126 & 131 & 136 & 137 & 97.2 & 138 & 133 & 122 & 131 & 132 & 145 & 134 & 146 & 47 & 151 & 143 \\
\hline $\mathrm{Y}$ & 18.9 & 20.2 & 19.8 & 20.6 & 20.4 & 19.7 & 23.6 & 19.9 & 16.6 & 16.9 & 19.1 & 20.3 & 19 & 17 & 20 & 42 & 21 & 19 \\
\hline $\mathrm{Zr}$ & 181 & 161 & 148 & 149 & 132 & 154 & 86.1 & 113 & 79.4 & 84.4 & 87.3 & 148 & 143 & 71 & 146 & 74 & 156 & 79 \\
\hline $\mathrm{La}$ & 37.71 & 34.88 & 32.84 & 33.44 & 30.07 & 30.80 & 36.95 & 27.79 & 24.36 & 26.26 & 26.19 & 33.88 & 28.88 & 24.46 & 33.80 & 36.91 & 33.68 & 25.65 \\
\hline $\mathrm{Ce}$ & 68.64 & 64.48 & 61.09 & 61.81 & 55.47 & 56.23 & 52.74 & 51.44 & 43.70 & 47.24 & 46.39 & 63.01 & 51.03 & 49.13 & 63.23 & 38.26 & 67.13 & 48.37 \\
\hline Pr & 7.92 & 7.45 & 7.02 & 7.15 & 6.55 & 6.53 & 7.84 & 6.00 & 4.79 & 5.18 & 5.25 & 7.30 & nd & nd & nd & nd & nd & nd \\
\hline $\mathrm{Nd}$ & 32.13 & 30.07 & 28.05 & 28.85 & 26.13 & 26.89 & 31.49 & 24.51 & 18.55 & 20.02 & 20.67 & 29.40 & 26.79 & 18.56 & 28.39 & 26.53 & 28.39 & 19.49 \\
\hline $\mathrm{Sm}$ & 7.14 & 6.65 & 6.26 & 6.38 & 5.82 & 6.37 & 7.17 & 5.37 & 4.09 & 4.38 & 4.66 & 6.41 & 6.98 & 4.69 & 6.64 & 6.62 & 6.89 & 4.67 \\
\hline $\mathrm{Eu}$ & 2.48 & 2.33 & 2.18 & 2.22 & 2.06 & 2.21 & 2.53 & 1.89 & 1.56 & 1.62 & 1.67 & 2.28 & 2.25 & 1.64 & 2.26 & 2.22 & 2.27 & 1.65 \\
\hline Gd & 6.52 & 6.21 & 5.64 & 5.78 & 5.36 & 5.69 & 7.08 & 5.27 & 4.26 & 4.49 & 4.60 & 6.08 & 6.58 & 4.78 & 6.20 & 7.93 & 6.41 & 4.75 \\
\hline $\mathrm{Tb}$ & 0.87 & 0.87 & 0.79 & 0.82 & 0.78 & 0.82 & 1.01 & 0.76 & 0.62 & 0.65 & 0.69 & 0.85 & nd & nd & nd & nd & nd & nd \\
\hline Dy & 4.31 & 4.57 & 4.20 & 4.37 & 4.18 & 4.30 & 5.37 & 4.03 & 3.36 & 3.58 & 3.83 & 4.49 & 4.35 & 3.43 & 4.39 & 5.54 & 4.43 & 3.75 \\
\hline Но & 0.68 & 0.75 & 0.71 & 0.74 & 0.70 & 0.71 & 0.90 & 0.70 & 0.59 & 0.62 & 0.68 & 0.75 & nd & nd & nd & nd & nd & nd \\
\hline Er & 1.51 & 1.81 & 1.71 & 1.75 & 1.67 & 1.68 & 2.17 & 1.76 & 1.45 & 1.57 & 1.67 & 1.82 & 1.72 & 1.53 & 1.70 & 2.54 & 1.77 & 1.61 \\
\hline $\mathrm{Tm}$ & 0.18 & 0.23 & 0.22 & 0.23 & 0.22 & 0.22 & 0.27 & 0.22 & 0.19 & 0.21 & 0.23 & 0.23 & nd & nd & nd & nd & nd & nd \\
\hline $\mathrm{Yb}$ & 1.01 & 1.36 & 1.27 & 1.34 & 1.29 & 1.20 & 1.65 & 1.34 & 1.18 & 1.25 & 1.33 & 1.42 & 1.11 & 1.12 & 1.25 & 1.61 & 1.30 & 1.28 \\
\hline $\mathrm{Lu}$ & 0.13 & 0.18 & 0.17 & 0.19 & 0.18 & 0.17 & 0.24 & 0.19 & 0.16 & 0.17 & 0.19 & 0.20 & 0.17 & 0.22 & 0.21 & 0.29 & 0.23 & 0.23 \\
\hline
\end{tabular}


normative nepheline (9\% to 25\%) with the exception indoleriticfacies showing normative hypersthene and weakly differentiated with mg numbers ranging from 0.38 to 0.70 . On the $\left(\mathrm{Na}_{2} \mathrm{O}+\mathrm{K}_{2} \mathrm{O}\right)$ versus $\mathrm{SiO}_{2}$ diagram [25], they spread from nephelinites, basanites, tephritesto basalts and hawaiites (Figure 4). The Quaternary lavas are relatively more siliceous (48\% - 52\%) and more sodic with $\mathrm{Na}_{2} \mathrm{O} / \mathrm{K}_{2} \mathrm{O}$ ratios values ranging between 2.06 and 8.70. They have low normative nepheline content $(1 \%$ and $8 \%)$ while doleritic facies show normative hypersthene. Their geochemical classification spreads from hawaiites, basalts totrachy-andesites. They are weakly differentiated with $\mathrm{mg}$ numbers ranging from 0.37 to 0.62 . The upper dolerites are more aluminous (17.92\%), more siliceous (52.17\%) and more ferrous (12.54\%) than other underlying basanites and dolerites.

The $\mathrm{Al}_{2} \mathrm{O}_{3}$ exhibits a positive correlation with the degree of silica saturation, whereas $\mathrm{MgO}, \mathrm{Fe}_{2} \mathrm{O}_{3}^{\mathrm{t}}, \mathrm{CaO}$, $\mathrm{Na}_{2} \mathrm{O}, \mathrm{P}_{2} \mathrm{O}_{5}, \mathrm{TiO}_{2}, \mathrm{MnO}$ and $\mathrm{Al}_{2} \mathrm{O}_{3} / \mathrm{CaO}$ are negatively correlated with the degree of silica saturation (Figure 5). These variations suggest differentiation processes dominated by clinopyroxene fractionation in the Cenozoic lavas.

\subsection{Trace Elements}

The concentrations of trace elements exhibit substantial differences between Tertiary and Quaternary rocks. The compatible elements (Cr, Ni, Co, Sc and V) are generally more abundant in Tertiary lavas, indicating various extents of fractionation of ferro-magnesian minerals whereas in the Quaternary lavas, the concentrations of these elements are less abundant.

Table 2. Sr and Nd isotopic datasof Cenozoic volcanic rocks ([6] unpublished datas).

\begin{tabular}{|c|c|c|c|c|}
\hline & Ref. Samples & $\left.{ }^{(87} \mathrm{Sr} /{ }^{86} \mathrm{Sr}\right) \mathrm{i}$ & ${ }^{143} \mathrm{Nd} /{ }^{144} \mathrm{Nd}$ & $\varepsilon \mathrm{Nd}$ \\
\hline \multirow[t]{3}{*}{ Quaternary volcanism } & DK11 & 0.702994 & 0.512935 & 5.79 \\
\hline & DK10 & 0.703196 & 0.512805 & 3.26 \\
\hline & DK4 & 0.703016 & 0.512919 & 5.48 \\
\hline \multirow[t]{6}{*}{ Tertiary volcanism } & DK18 & 0.704076 & 0.51289 & 4.92 \\
\hline & DK13 & 0.703254 & 0.512971 & 6.5 \\
\hline & DK14 & 0.704636 & 0.512923 & 5.56 \\
\hline & DK1 & 0.703191 & 0.512932 & 5.74 \\
\hline & DK3 & 0.703064 & 0.512898 & 5.07 \\
\hline & DK8 & 0.702908 & 0.51293 & 5.7 \\
\hline
\end{tabular}

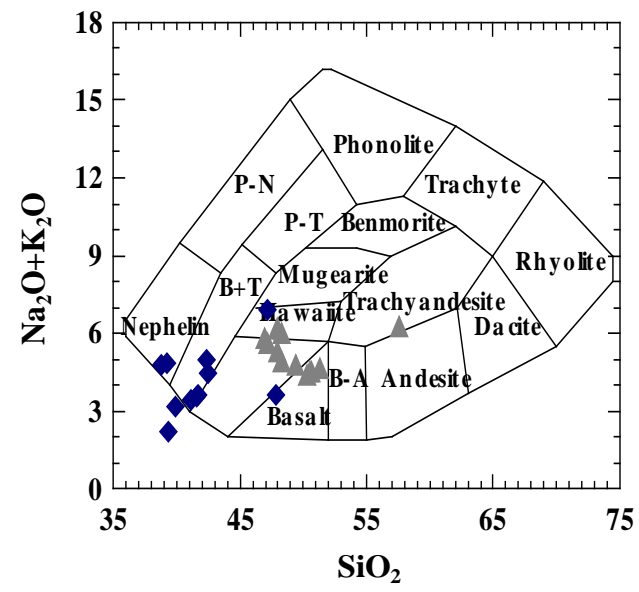

Figure 4. Distribution of Tertiary lavas (diamond) and Quaternary lavas (triangle) on the alkali versus silica diagram [25]. 

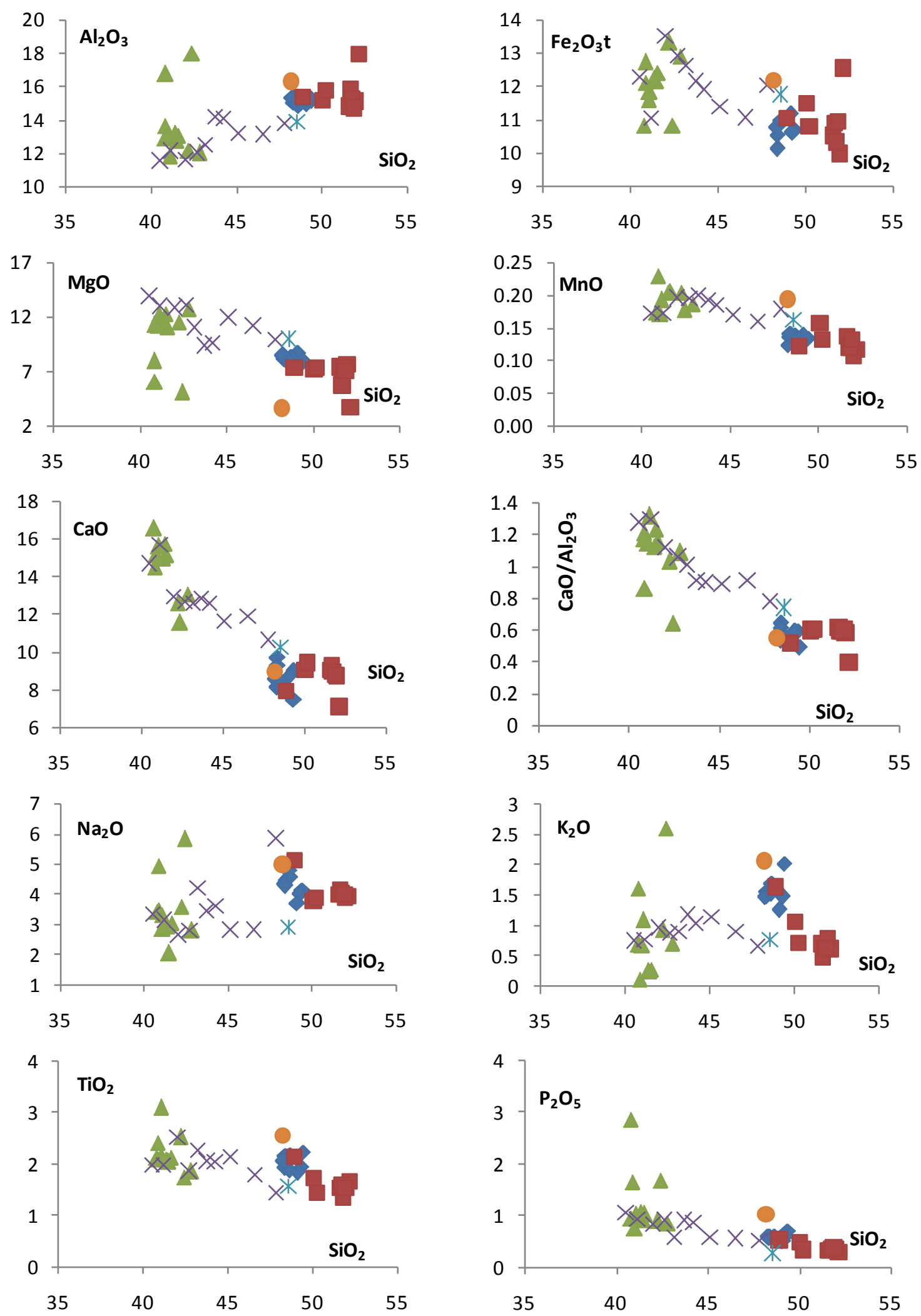

Figure 5. Plots of $\mathrm{SiO}_{2}$ vs oxides for the Cenozoic volcanic rocks: diamond (Quaternary basanites), square (Quaternary dolerite), triangle (Tertiary nephelinites), cross (Tertiary basanites), asterisk (Tertiary dolerites), round (Tertiary gabbro). 
The thorium considered as a strongly hygromagmaphile element shows a positive correlation with the incompatible elements and a negative correlation with the compatible elements (Figure 6). Compatible elements exhibit small variations in the Quaternary rocks whereas the values observed in the Tertiary rocks are notably different according to thorium contents.

The distribution of the rare-earth elements (REE) is typical of alkaline lavas, with generally strong light-REE
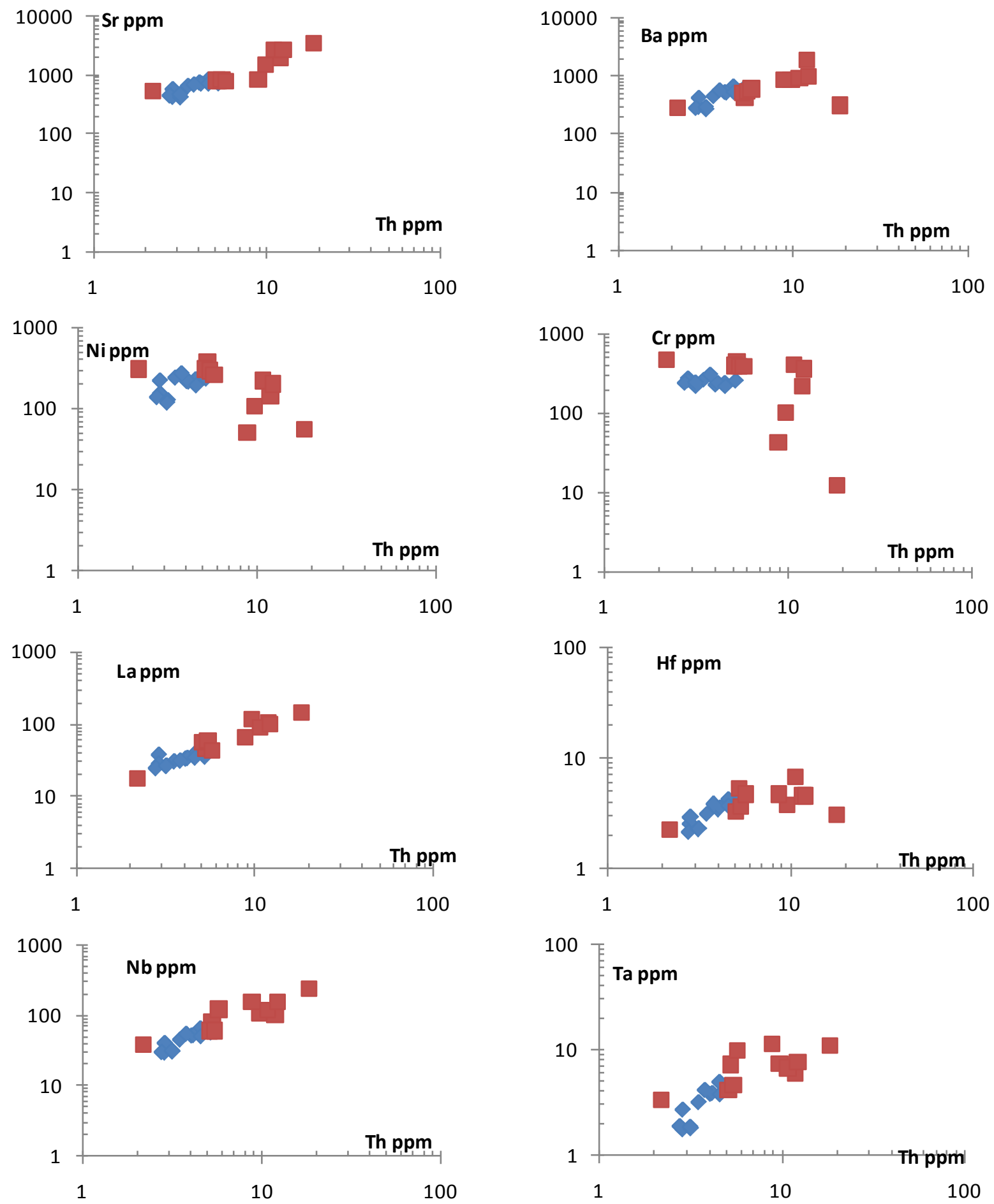

Figure 6. Selected compatible and incompatible elements as function of thorium concentrations of Cenozoic volcanic rocks: diamond (Quaternary rocks), square (Tertiary rocks). 
(LREE) enrichments defining regularly sloping patterns without significant Eu-anomaly (Figure 7). Tertiary lavas are the most enriched with high values of $\mathrm{La}_{N}(120$ - 320) and large variations of $\mathrm{La} / \mathrm{Yb}(28-60)$, whereas the Quaternary spectra are much less enriched and more homogeneous with $\mathrm{La}_{\mathrm{N}}<100$ and $\mathrm{La} / \mathrm{Yb}$ varying from 20 to 27.

The good correlations formerly observed between thorium and trace elements and the parallelism of the representative REE patterns suggest in first approximation a common magmatic source. The Y/N bratios $(0.17$ $0.78)$ and $(\mathrm{La} / \mathrm{Yb})_{\mathrm{N}}$ values $\left(8.63\right.$ - 34.98) are closed to those alkali basalts where $\mathrm{Y} / \mathrm{Nb}<1$ and $(\mathrm{La} / \mathrm{Yb})_{\mathrm{N}}>12$.

The spider-diagrams (Figure 8) show a continuous enrichment from $\mathrm{Yb}$ to $\mathrm{Nb}$ followed by a sharp (in Tertiary lavas) or moderate (in Quaternary lavas) decrease of $\mathrm{K}$ and Rbto normalized concentrations. The characters as the $\mathrm{Nb}$ culminating patterns, the negative K-anomaly and the LILE enrichment, with low $\mathrm{La} / \mathrm{Nb}, \mathrm{Rb} / \mathrm{Nb}$, $\mathrm{K} / \mathrm{Nb}, \mathrm{Th} / \mathrm{Nb}, \mathrm{Zr} / \mathrm{Nb}$ and $\mathrm{Ba} /$ Laratios make them similar to oceanic islands basalts, particularly HIMU-type oceanic basalts [26].

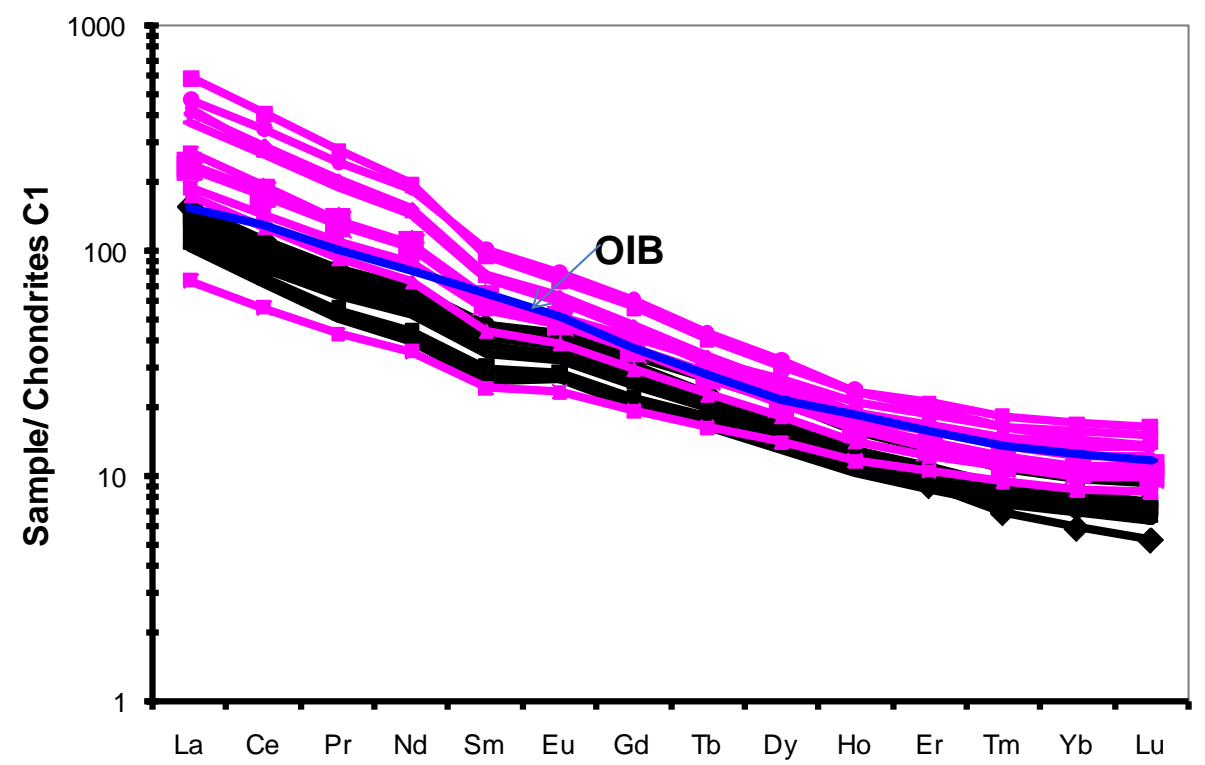

Figure 7. Chondrite-normalized REE patterns of the Cenozoic volcanic rocks: pink (Tertiary rocks), black (Quaternary rocks). Normalizing C1 and OIB (oceanic islands basalts) values from [32].

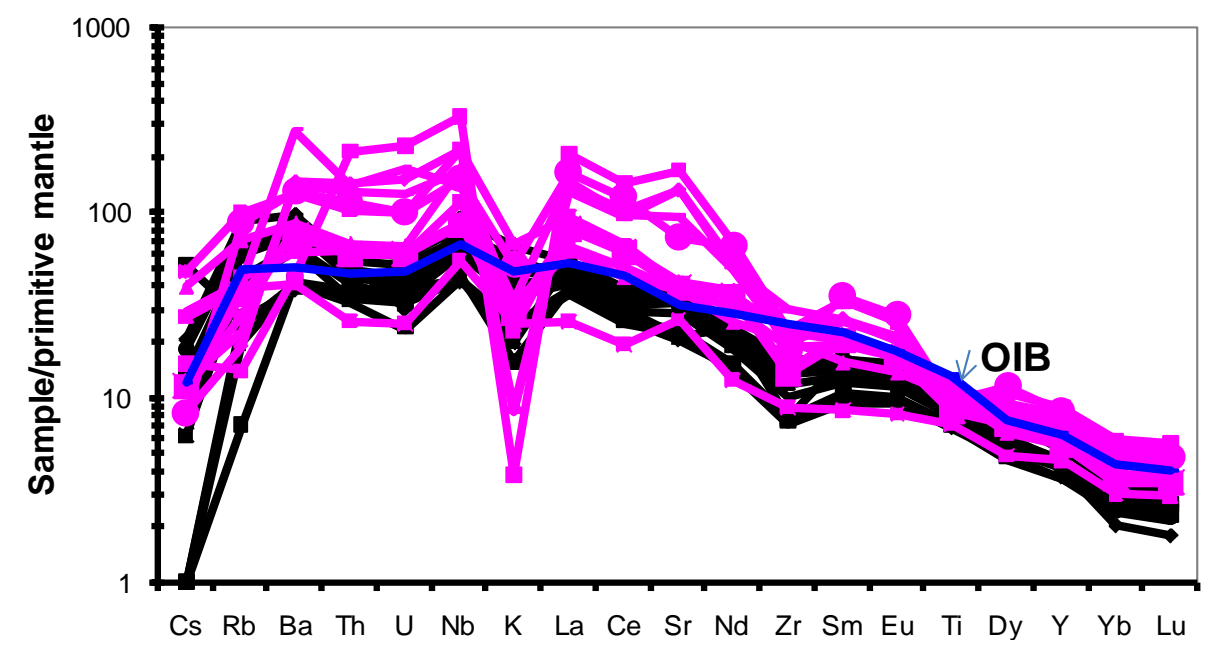

Figure 8. Primitive mantle-normalized trace elements abundance of the Cenozoic volcanic rocks: pink (Tertiary rocks), black (Quaternary rocks). Normalizing primitive mantle and OIB (oceanic islands basalts) values from [32]. 
The enriched character of the Tertiaryrocks compared to the Quaternary rocks could be explained by a subcontinental enriched mantle source or an interaction of metasomatic fluids with the magmatic source just before the melting. The prophyritic (or pegmatitoids) nephelinites which are significantly more enriched than other Tertiary and Quaternarylavas, contained apatite, amphibole, biotite and calcite interpreted as an early interaction of magma with fluid phases rich in $\mathrm{H}_{2} \mathrm{O}$ and $\mathrm{CO}_{2}$ ([19] [27] [28]). The hypothesis of a metasomatized source could be explained by the enrichment of $\mathrm{K}, \mathrm{Rb}, \mathrm{Ba}$, Sr, LREE and HFSE which are highly solublein aqueous solutions according to [29]-[31]. Moreover the low values of Hf/Sm ratiosin Tertiary (0.21 to 0.63) and Quaternary (0.36 to 0.61$)$ lavas compared to those of primitive mantle (0.695) of [32] are in accordance with metasomatic fluids rich in carbonates [33] [34]. These results are in agreement with the detailed studies on mantle xenoliths and lavas of the Cenozoic Cape Verde archipelagoes volcanism which have shown kimberlitic or carbonatitic melts at the origin of metasomatic fluids [35] [36].

\subsection{The Isotopic Results}

The Nd and Sr isotopes [6], (unpubl. data) of nine samples from the Tertiary and Quaternary volcanism cover the total duration of the volcanic activity and are representative of the groups previously defined (Table 3 ).

All the Tertiarylavas are characterized by low radiogenic Srvalues $(0.703064-0.703254)$ and positive $\varepsilon \mathrm{Nd}$ $(+5.07$ to +6.5$)$ excepted porphyritic (or pegmatitoids) nephelinites which are more radiogenic Sr $(0.704105$ $0.704652)$ for comparable $\varepsilon \mathrm{Nd}$ values (+4.92 to +5.56). Moreover, the Quaternary lavas are slightly less Sr radiogenic (0.70299 to 0.70302$)$ with positive $\varepsilon \mathrm{Nd}(+5.48$ to +5.79$)$. The quaternary rock DK 10 differs from others, with more radiogenic $\mathrm{Sr}(0.70320)$ and less radiogenic $\mathrm{Nd}(\mathrm{ENd}=+3.26)$. This high ${ }^{87} \mathrm{Sr} /{ }^{86} \mathrm{Sr}$ value could be interpreted as reflecting the effects of alteration which modified the magmatic Sr isotopic signatures.

The weak radiogenic strontium and more radiogenic neodymium with positive $E N d(+3.26$ to +6.5$)$ are intermediary between DMM and HIMU OIB-type suggesting an origin from a mantle less depleted than those of ocean ridges basalts (Figure 9). These results are fairly similar of those obtained in the Cape Verde Archipelagoes [38].

Several lines of evidence indicate that the studied volcanic rocks did not suffer from significant crustal contamination. The existence of mantle xenoliths and the lack of differentiated lavas suggest that the magmas were not retained long enough within the crust to be contaminated. A possible contamination by lithospheric crust may be considered to explain the increase of silica contents in Quaternarylavas. According to [39], the average $\mathrm{Nb} / \mathrm{U}$ values for the MORB and OIBis fixed at $47 \pm 10$ and any increase in this ratio associated with the decrease of uranium values, could be interpreted as the result of acrustal influence. The latest magmatic manifestations during the Tertiary period (Diack pluton) which outcrop at the eastern parts of the volcanic province have relatively high $\mathrm{Nb} / \mathrm{U}$ ratios (64 - 88) compared to the Quaternary lavas (63 - 106) which are localized at the western parts of the basin at the front of the Atlantic Ocean. In addition, these rocks from Diack pluton have higher values of $\mathrm{Zr} / \mathrm{U}(119$ - 288) and $\mathrm{Nb} / \mathrm{Th}(11$ - 21) than the other rocks which show no crustal contamination as evidenced by their low Nb/U (45 - 51), Zr/U (53 - 95) and Nb/Th (11 - 12) values (Figures 10(a)-(c)).

\section{Discussions and Conclusions}

The Cenozoic volcanism of the Cap-Vert peninsula is marked by a magmatic activity spread over $35 \mathrm{Ma}$, from Late Eocene up to Middle Pleistocene. It belongs to the last magmatic episode known in the western and the northwestern parts of the West Africa during the Permian up to the Quaternary. It is characterized by different periods of activities where no age progression is related to the geographic localization of outcrops as it is shown on the map (cf. Figure 3(a)). This volcanism is moderately to strongly alkaline with sodic affinity and weakly differentiated during his activity. The lack of any evolved products could be explained by a short time residence in the magmatic chamber. However, the Quaternary lavas are more sodic, less alkaline and relatively more differentiated in comparison to the Tertiary one.

The Cap-Vert peninsula volcanism is previously interpreted as anintra-plate volcanism associated with the migration of hot spot activities [9] [10]. On the basis of K-Ar ages data, the Cap-Vert peninsula volcanism is contemporary to the Cape Verde and Canary archipelagoes volcanism by their ages of their manifestations and their geodynamic significance [6].

In fact, the evolution of the Cap-Vert peninsula volcanism is characterized by different periods of magmatic activities (Table 3). The early ages of this manifestation are recorded around the Middle Eocene specifically 
Table 3. K-Ar ages of the Cap-Vert peninsula volcanic rocks and comparison with Cape Verde (CVA) [38] [41]-[43] and Canary (CANA) [44] [45] archipelagoes duration of volcanism. Samples with asterisk $(*)$ are from the PASMI (unpublished report 2009) and the others from [6].

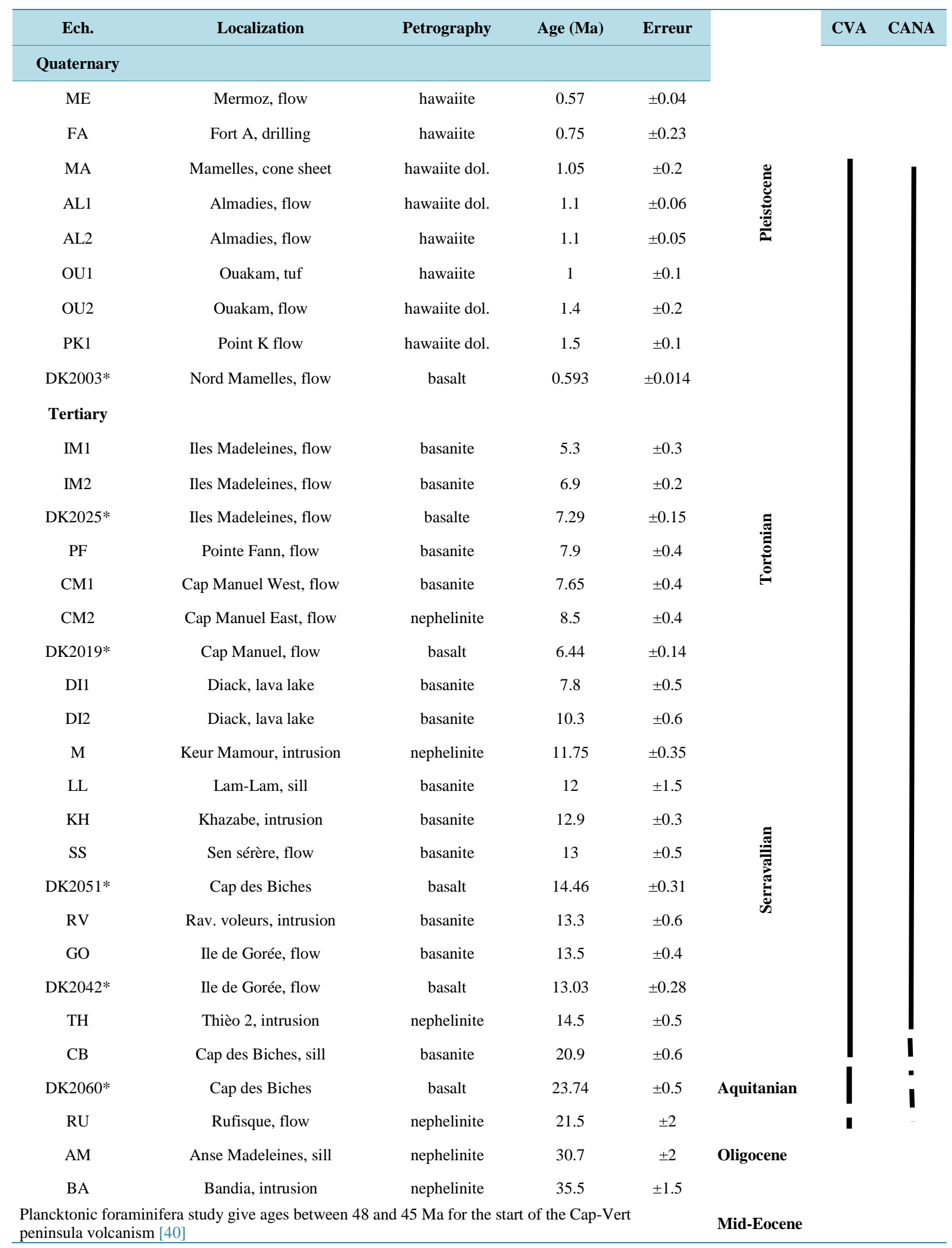




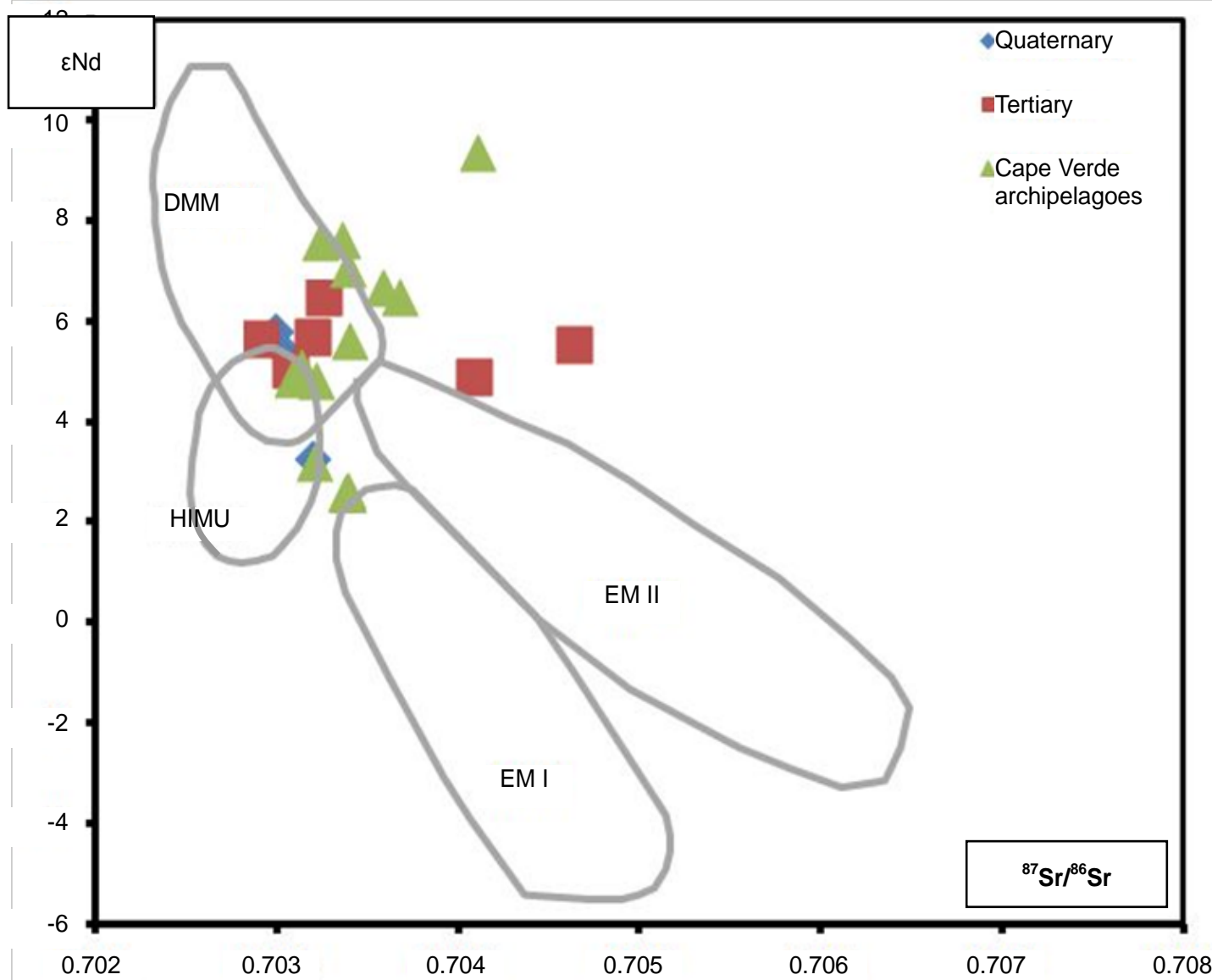

Figure 9. Distribution of Tertiary (III) and Quaternary (IV) volcanic rocks in the ${ }^{87} \mathrm{Sr} /{ }^{86} \mathrm{Sr}$ vs $\varepsilon$ Nd diagram (datas of Cape Verde archipelagoes from [38]). The DMM, HIMU, EMI and EMII domains are defined by [37].

between 48 and 45 Ma by [40]. The first manifestation of this volcanism occurred during Oligocene (35 to 20 $\mathrm{Ma}$ ) and is marked by magmatic intrusions in the Paleocene limestones. The peak of this volcanism is recorded during the Miocene (14 to $5 \mathrm{Ma}$ ). It is widely spread in Dakar and the eastern parts of the peninsula, up to Thiès area. The last manifestation is dated during the Pleistocene (1.4 to $0.5 \mathrm{Ma}$ ) and is only localized in the Head of Cap-Vert peninsula known as the Mamelles volcano.

The ages recorded in the Cape Verde and Canary Archipelagoes volcanism are relatively more recent in comparison to those observed in the Cap-Vert peninsula. In the Cape Verde Archipelago, the first events of the Cenozoic volcanism are dated around 25.6 Ma in the Old Eruptive Complex of Sal Island [38] [41]-[43]; the paroxysm of his activity was reached during the Miocene (16.3 and 5.6 Ma) and continued during the Quaternary age (1.06 to 0.6 Ma). In the Canary Archipelago, the first manifestations are recorded around 20 Main Lanzarote and Fuerteventura Islands; this activity continued in Gran Canary, Tenerife and La Gomera Islands between 15 to $8 \mathrm{Ma}$ and lately in Palma and HierroIslands [44] [45].

In the Senegalo-Mauritanian basin, an early plutonicalkaline phase took place in the early Maastrichtian. Then the Cenozoic volcanism began in the Cap-Vert peninsula at the limit Eocene-Oligocene and continued up to 0.6 Ma, while it was still active in Cape Verde (Fogo Island) and Canary Islands (Tenerife and La Palma).

This Cenozoic volcanism is connected to the uplift of lithospheric blocks in response to the Alpine tectonic events occurring in the northwestern Africa. In the Cape Verde and most of the Canary Archipelagoes, the volcanism was at first submarine and becoming subaerial later while in the Cap-Vert peninsula and possibly the easternmost Canary Islands, the volcanism sets on a transitional crust [4] [46] [47].

In fact, in all these three provinces, the Cenozoic magmatism is strongly alkaline and mainly undersatured, with olivine nephelinites and olivine-melilite nephelinites being fairly common. However, the volcanic series of 

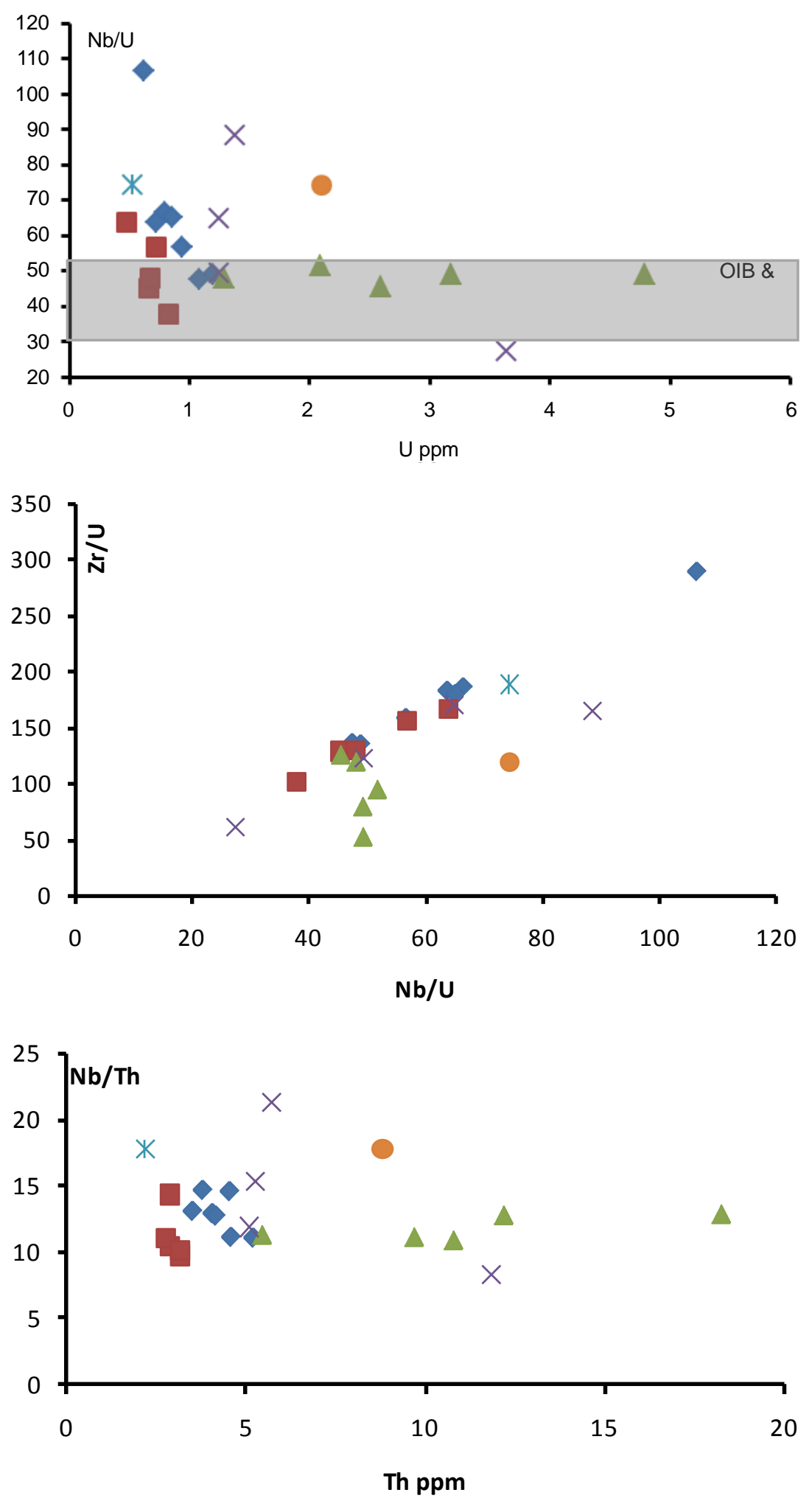

Figure 10. (a), (b), (c) - Incompatible elements ratios evolutions in the Cenozoic volcanism of Cap Vert peninsula: diamond (Quaternary basanites), square (Quaternary dolerite), triangle (Tertiary nephelinites), cross (Tertiary basanites), asterisk (Tertiary dolerites), round (Tertiary gabbro). The shaded portion corresponds to the $\mathrm{Nb} / \mathrm{U}$ ratios values $47 \pm 10$ of OIB and MORB values [39]. 


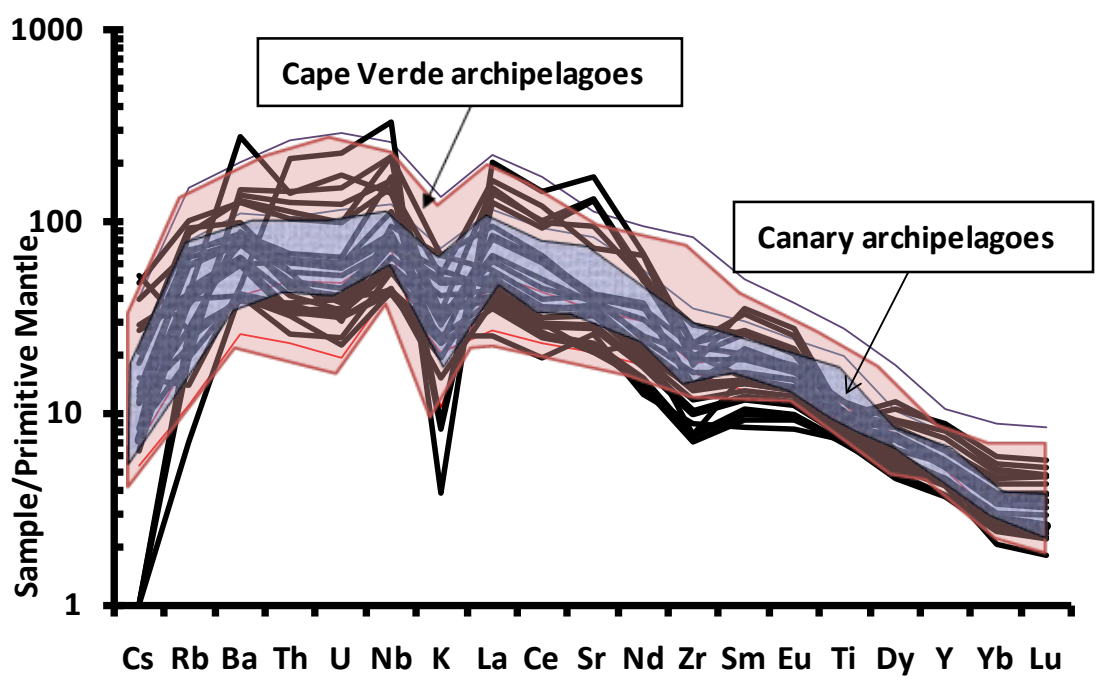

Figure 11. Comparisons of incompatible trace elements concentrations of mafic lavas from the Cape Verde archipelagoes (pink field defined by $\mathrm{n}=26,5<\mathrm{MgO}>10 \%$, [43]. and Canary archipelagoes (blue field defined by $\mathrm{n}=25, \mathrm{MgO}>8 \%$, [48]. with the Cap Vert peninsula (present study) normalized to the primitive mantle compositions [32].

the Cap-Vertpeninsula remain less differentiated in comparison to the Cape Verde and Canary archipelagoes ones, where mildly alkaline to transitional series are reported [38] [43]. In spite of these differences, they exhibit similar geochemical characteristics as it is shown by the variations of trace elements (Figure 11). Dy/Yb (2.45 4.24) and $\mathrm{Zr} / \mathrm{Hf}(33$ - 48) ratios are close enough to those obtained in Canary Island (2.8 - 3.6 and 24 - 39) [44] [48] and in Cape Verde archipelago (2.3 - 4.06 and 35 - 69) [41]. Furthermore radiogenic isotopic compositions are characterized by low ${ }^{87} \mathrm{Sr} /{ }^{86} \mathrm{Sr}$ values and high ${ }^{143} \mathrm{Nd} /{ }^{144} \mathrm{Nd}$, suggesting that all these Cenozoic volcanic provinces share fairly a common sub-lithospheric mantle source.

The heterogeneity of the Cenozoic volcanism source in the Cape Verde and the Canary Archipelagoes is widely discussed [38] [43] [48]. In the Cape Verde archipelagoes, this heterogeneity is marked by the contribution of HIMU and DMM component in the magmatic sources of the Northern Islands as it is shown by their high ${ }^{143} \mathrm{Nd} /{ }^{144} \mathrm{Nd}$ values comparable of those observed in Cap-Vert peninsula volcanism, whereas the EM1 component is restricted to the Southern Islands (CVS) sources where the ${ }^{143} \mathrm{Nd} /{ }^{144} \mathrm{Nd}$ values are lower.

The sources of this volcanism are related to the sub-lithospheric plume channeling processes which are beginning at the Triassic-Jurassic stage in the realm of the future central Atlantic Ocean. This process has continued throughout the Cenozoic stage leading to alkaline magmatism along a north-northeast trend with decreasing ages from the Cape Verde archipelago up to Europe [49]. Such a model could explain the early manifestations of this volcanism in the Cap-Vert peninsula as compared to Cape Verde and Canary Archipelagoes.

\section{Acknowledgements}

Thank you to Professors Gilbert Crévola and Christian Moreau for supplementary analytical data for the production of this document and to Umberto Cordani and Pierre Barbey for help to improve the presentation of the document.

\section{References}

[1] Meagher, L.J., Ruffman, A. and Steward, W. (1977) Contribution à la géophysique du plateau continental du Sénégal et de la Gambie. Afrique de l’Ouest. Baffin, 38-40.

[2] Chautard, J. (1907) Les roches éruptives de la presqu’île du Cap Vert (Sénégal). Bull. Soc. Géol. Fr., 424-440.

[3] Combier, M. (1935) Carte Géologique de Dakar (notice explicative). Bull. Com. Et. Hist. Et Sc. AOF, série B, n¹.

[4] Bellion, Y. and Crévola, G. (1991) Cretaceous and Cainozoic Magmatism of the Senegal Basin (West Africa): A Review. In: Kampunzu, A.B. and Lubala, R.T., Eds., Magmatism in Extensional Structural Settings: The Phanerozoic Af- 
rican Plat, Springler Verlag, Berlin, 189-208. http://dx.doi.org/10.1007/978-3-642-73966-8_8

[5] Guiraud R., Bellion Y., Benkhelil J. and Moreau C. (1987) Post-Hercynian Tectonics in Northern and Western Africa. Journal of Geology, 22, 433-466.

[6] Crévola, G., Cantagrel, G.M. and Moreau, C. (1994) Le volcanisme cénozoïque de la presqu'île du Cap-Vert (Sénégal): Cadre chronologique et géodynamique. Bull. Soc. Geol. Fr., t. 165, 437-446.

[7] Rocchi, S., Mazotti, A., Morroni, M., Pandolfi, P., Giusepp, B., di Biase, D., Federici, F. and Lo, P.G. (2007) Detection of Miocene Saucer-Shaped Sills (Offshore Senegal) via Integrated Interpretation of Seismic, Magnetic, and Gravimetric Data.Terra Nova, 19, 232-239. http://dx.doi.org/10.1111/j.1365-3121.2007.00740.x

[8] Hansen, D.M., Redfern, J., Federici, F., di Biase, D. and Guiseppe, B. (2008) Miocene Igneous Activity in the Northern Sub-Basin, Offshore Senegal, NW Africa. Marine and Petroleum Geology, 25, 1-15. http://dx.doi.org/10.1016/j.marpetgeo.2007.04.007

[9] Lô, P.G., Dia, A. and Kampunzu, A.B. (199) Cenozoic Volcanism in the Western Senegal and Its Relationship to the Opening of the Central Atlantic Ocean. Tectonophysics, 209, 281-291. http://dx.doi.org/10.1016/0040-1951(92)90035-5

[10] Mellet, O. (1993) Etude des mécanismes de mise en place et de la pétrogenèse du volcano-sédimentaire quaternaire de la presqu'île du Cap-Vert (Sénégal occidental). Mem. DEA Geosciences, INPL, Nancy, 135.

[11] Bellion, Y. (1987) Histoire géodynamique post-paléozoïque des bassins d'Afrique de l’Ouest. Thèse Etat, University of Avignon, Avignon, 306 p.

[12] de Spengler, A., Castelain, J., Cauvin, J. and Leroy, M. (1966) Le bassin Secondaire-Tertiaire du Sénégal. In: par Reyre, D., Ed., Bassins sédimentaires du littoral africain, $1^{\text {ère }}$ partie: Littoral Atlantique, Association des Services Géologiques Africaines, Paris, 80-94.

[13] Ligier, J.L. (1980) Structure profonde du basin côtier sénégalo-mauritanien. Interprétation des données gravimétriques et magnétiques. Thèse 3éme cycle, Trav. Lab. Sci. Terre, Marseille, B, 116, 158 p.

[14] Ritz, M., Bellion, Y. and Flicoteaux, R. (1987) Magnetelluric Soundings and the Geological Structure and Tectonics of the Senegalo-Mauritanian Basin in the Northern Senegal, West Africa. Tectonics, 6, 395-409.

[15] Bertrand, H. (1991) The Mesozoic Tholeiitic Province of Northwest Africa: A Volcano-Tectonic Record of the Early Opening of Central Atlantic. In: Kampunzu, A.B. and Lubala, R.T., Eds., Magmatism in Extensional Structural Settings: The Phanerozoic African Plate, Springler Verlag, Berlin, 147-188. http://dx.doi.org/10.1007/978-3-642-73966-8_7

[16] Seibold, E. and Hinz, K. (1974) Continental Slope Construction and Destruction, West Africa. In: Burk, C.A. and Drake, C.L., Eds, The Geology of Continental Margins, Springer Verlag, Berlin, 179-196. http://dx.doi.org/10.1007/978-3-662-01141-6_13

[17] Gomez, R. and Barusseau, J.P. (1984) Dispositions des formations post-éocènes de la marge continentale sénégalaise. Bulletin de la Société Géologique de France, 26, 1107-1116.

[18] Lompo, M. (1987) Méthodes et étude de la fracturation et des filons. Exemple de la région du Cap-Vert (Sénégal). Mémoire de Diplôme d’Etudes Approfondies (D.E.A), Dakar, 59 p.

[19] Crévola, G. (1978) Sills, dykes, et pipes de tufs volcaniques bréchiques fluidifiés dans la presqu'île du Cap-Vert (Sénégal). Comptes Rendus Sommaires de la Société Géologique de France, 3, 135-139.

[20] Gorodiski, A. (1952) Notice explicative de la carte géologique du Sénégal à 1/20000 (feuilles Ouakam et Dakar). Bulletin de la Direction des Mines de l'Afrique Occidentale Française (AOF) (Dakar), 10, 5-57.

[21] Bellion, Y. and Guiraud, R. (1984) Le bassin sédimentaire du Sénégal. Synthèse des connaissances actuelles. In: Bureau des Recherches Géologiques et Minières (BRGM) et Direction des Mines et de la Géologie (DMG), Dakar, Eds., Plan minéral de la république du Sénégal, Dakar, 4-63.

[22] Ndiaye, A. (2002) Etude lithologique et pétrographique des coulées de laves quaternaires de la presqu’île du Cap-Vert (Sénégal). Mém. DEA Fac., Sciences et Techniques Univ. Ch. A. DIOP, Dakar.

[23] Carignan, J., Hild, P., Mévelle, G., Morel, J. and Yeghicheyan, D. (2001) Routine Analyses of Trace Elements in Geological Samples Using Flow Injection and Low Pressure On-Line Liquid Chromatography Coupled to ICP-MS: A Study of Reference Materials BR, DR-N, UB-N, AN-G and GH. Geostandards Newsletter, 25, 187-198. http://dx.doi.org/10.1111/j.1751-908X.2001.tb00595.x

[24] Pin, C., Briot, D., Bassin, C. and Poitrasson, F. (1994) Concomitant Separation of Strontium and Samarium-Neodymium for Isotopic Analysis in Silicates Samples, Based on Specific Extraction Chromatography. Analytica Chimica Acta, 298, 209-217.

[25] Cox, K.G., Bell, J.D. and Pankhurst, R.J. (1979) The Interpretation of Igneous Rocks. George Allen \& Unwin, London, 450 p. http://dx.doi.org/10.1007/978-94-017-3373-1 
[26] Weaver, B.L. (1991) The Origin of Ocean Island Basalt End-Member Compositions: Trace Element and Isotopic Constraints. Earth and Planetary Science Letters, 104, 381-397. http://dx.doi.org/10.1016/0012-821X(91)90217-6

[27] Combier, M. (1952) Note sur les pegmatitoïdes de Gorée et de l’Ile aux Serpents. Dakar, Bulletin de la Direction des Mines de l'Afrique Occidentale Française (AOF), 10, 55-108.

[28] Crévola, G. (1975) Le volcanisme tertiaire et quaternaire de la presqu'île du Cap-Vert (Sénégal). Bulletin de l'Association pour l'Avancement des Sciences Naturelles au Sénégal (AASNS), 40, 19-39.

[29] Loughnan, F.C. (1969) Chemical Weathering of the Silicate Minerals. Elsevier, New York.

[30] Wright, T.L. (1984) Origin of Hawaiian Tholeiite: A Metasomatic Model. Journal of Geophysical Research, 89, 32333252. http://dx.doi.org/10.1029/JB089iB05p03233

[31] Tatsumi, Y., Hamilton, D.L. and Nesbitt, R.W. (1986) Chemical Characteristics of Fluids Phase Released from a Subducted Lithosphere and Origin of Arc Magmas: Evidence from High Pressure Experiments ad Natural Rocks. Journal of Volcanology and Geothermal Research, 29, 293-309. http://dx.doi.org/10.1016/0377-0273(86)90049-1

[32] Sun, S.S. and McDonough, W.F. (1989) Chemical and Isotopic Systematics of Oceanic Basalts: Implications for Mantle Compositions and Processes. In: Saunders, A.D. and Norry, M.J., Eds., Magmatism in the Oceanic Basins, Geological Society, Special Publications, London, 42, 313-345.

[33] Hamilton, D.L., Bedson, P. and Esson, J. (1989) The Behaviour of Trace Elements in the Evolution of Carbonatites. In: Bell, K., Ed., Carbonatites: Genesis and Evolution, Unwin Hyman, Boston, 405-427.

[34] Dostal, J., Zhi, X.C., Muehlenbachs, K., Dupuy, C. and Zhai, M.Z. (1991) Geochemistry of Cenozoic Alkali Lavas from Shandong Province, Eastern China. Geochemical Journal, 25, 1-16. http://dx.doi.org/10.2343/geochemj.25.1

[35] Bonadiman, C., Beccaluva, L., Coltorti, M. and Siena, F. (2005) Kimberlite-Like Metasomatism and “Garnet Signature” in Spinel-Peridotite Xenoliths from Sal, Cape Verde Archipelago: Relics of a Subcontinental Mantle Domain within the Atlantic Ocean Lithosphere? Journal of Petrology, 46, 2465-2493. http://dx.doi.org/10.1093/petrology/egi061

[36] Martins, S., Mata, J., Munhá, J., Mendes, M.H., Maerschalk, C., Caldeira, R. and Mattielli, N. (2010) Chemical and Mineralogical Evidence of the Occurrence of Mantle Metasomatism by Carbonate-Rich Melts in an Oceanic Environment (Santiago Island, Cape Verde). Mineralogy and Petrology, 99, 43-65. http://dx.doi.org/10.1007/s00710-009-0078-X

[37] Chauvel, C. and White, W.M. (1998) Géochimie isotopique du volcanisme océanique actuel. Identification des grands domaines mantelliques sources. In: Hagemann et Treuil, Eds., Introduction à la Géochimie et ses Applications, CEA, Manson T.1, Chap. 5, 269-286.

[38] Torres, P., Silva, L.C., Munha, J., Caldeira, L., Mata, J. and Tassinari, C. (2010) Petrology and Geochemistry of Lavas from Sal Island: Implications for the Variability of the Cape Verde Magmatism. Cominucacoes Geologicas, 97, 35-62.

[39] Hofmann, A.W., Jochum, K.P., Seufert, M. and White, W.M. (1986) Nb and Pb in Oceanic Basalts: New Constraints on Mantle Evolution. Earth and Planetary Science Letters, 79, 33-45. http://dx.doi.org/10.1016/0012-821X(86)90038-5

[40] Sarr, R., Ndiaye, P.M., Niang-Diop, I. and Guéye, M. (2000) Datation par les foraminifères planctoniques d'une activité volcanique d’âge Lutétien à Toubab Dialao (Sénégal occidental). Bulletin de la Société Géologique de France, 171, 197-205.

[41] Michell, J.G., Le Bas, M.J., Zielonka, J. and Furnes, H. (1983) On Dating the Magmatism of Maio, Cape Verde Islands. Earth and Planetary Science Letters, 64, 61-76. http://dx.doi.org/10.1016/0012-821X(83)90053-5

[42] Plesner, S., Holm, P.M. and Wilson, J.R. (2002) ${ }^{40} \mathrm{Ar}-{ }^{39} \mathrm{Ar}$ Geochronology of Santo Antão, Cape Verde Islands. Journal of Volcanology and Geothermal Research, 120, 103-121. http://dx.doi.org/10.1016/S0377-0273(02)00367-0

[43] Holm, P.M., Wilson, J.R., Christensen, B.P., Hansen, L., Hansen, S.L., Hein, K.M., Mortensen, A.K., Pedersen, R., Plesner, S. and Runge, M.K. (2006) Sampling the Cape Verde Mantle Plume: Evolution of the Melt Compositions on Santo Antão, Cape Verde Islands. Journal of Petrology, 47, 145-189.

[44] Hoernle, K., Tilton, G. and Schmincke, H.U. (1991) Sr-Nd-Pb Isotopic Evolution of Gran Canaria: Evidence for Shallow Enriched Mantle beneath the Canary Islands. Earth and Planetary Science Letters, 106, 44-63. http://dx.doi.org/10.1016/0012-821X(91)90062-M

[45] Carracedo, J.C., Day, S., Guillou, H., Rodriguez Badiola, E., Canas, J.A. and Perez Torrado, F.J. (1998) Hotspot Volcanism Close to a Passive Continental Margin: The Canary Islands. Geological Magazine, 135, 591-604. http://dx.doi.org/10.1017/S0016756898001447

[46] De Paepe, P., Klerkx, J., Hertogen, J. and Plinke, P. (1974) Oceanic Tholeiites on the Cape Verde Islands: Petrochemical and Geochemical Evidence. Earth and Planetary Science Letters, 22, 347-354. http://dx.doi.org/10.1016/0012-821X(74)90144-7

[47] Arana, V. and Ortiz, R. (1991) The Canary Islands: Tectonics, Magmatism and Geodynamic Framework. In: Kam- 
punzu, A.B. and Lubala, R.T., Eds., Magmatism in Extentional Structural Settings (The Phanerozoic African Plate), Springer-Verlag, Barcelone, 209-563.

[48] Lundstrom, C.C., Hoernle, K. and Gill, J. (2003) U-Series Disequilibria in Volcanic Rocks from the Canary Islands: Plume versus Lithospheric Melting. Geochimica et Cosmochimica Acta, 67, 4153-4177. http://dx.doi.org/10.1016/S0016-7037(03)00308-9

[49] Oyarzun, R., Doblas, M., Lopez-Ruiz, J. and Cebrà, J.M. (1997) Opening of the Central Atlantic and Asymmetric Mantle Upwelling Phenomena: Implications for Long-Lived Magmatism in Western North Africa and Europe. Geology, 25, 727-730. http://dx.doi.org/10.1130/0091-7613(1997)025<0727:OOTCAA >2.3.CO;2 
Scientific Research Publishing (SCIRP) is one of the largest Open Access journal publishers. It is currently publishing more than 200 open access, online, peer-reviewed journals covering a wide range of academic disciplines. SCIRP serves the worldwide academic communities and contributes to the progress and application of science with its publication.

Other selected journals from SCIRP are listed as below. Submit your manuscript to us via either submit@scirp.org or Online Submission Portal.
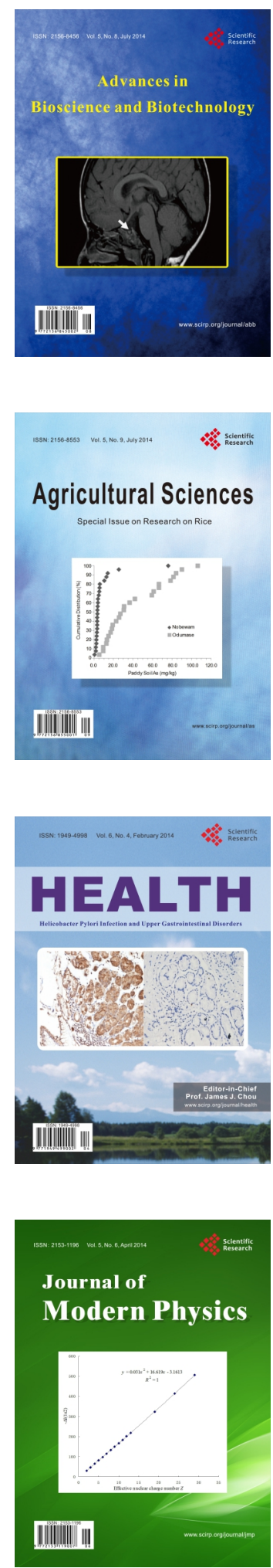
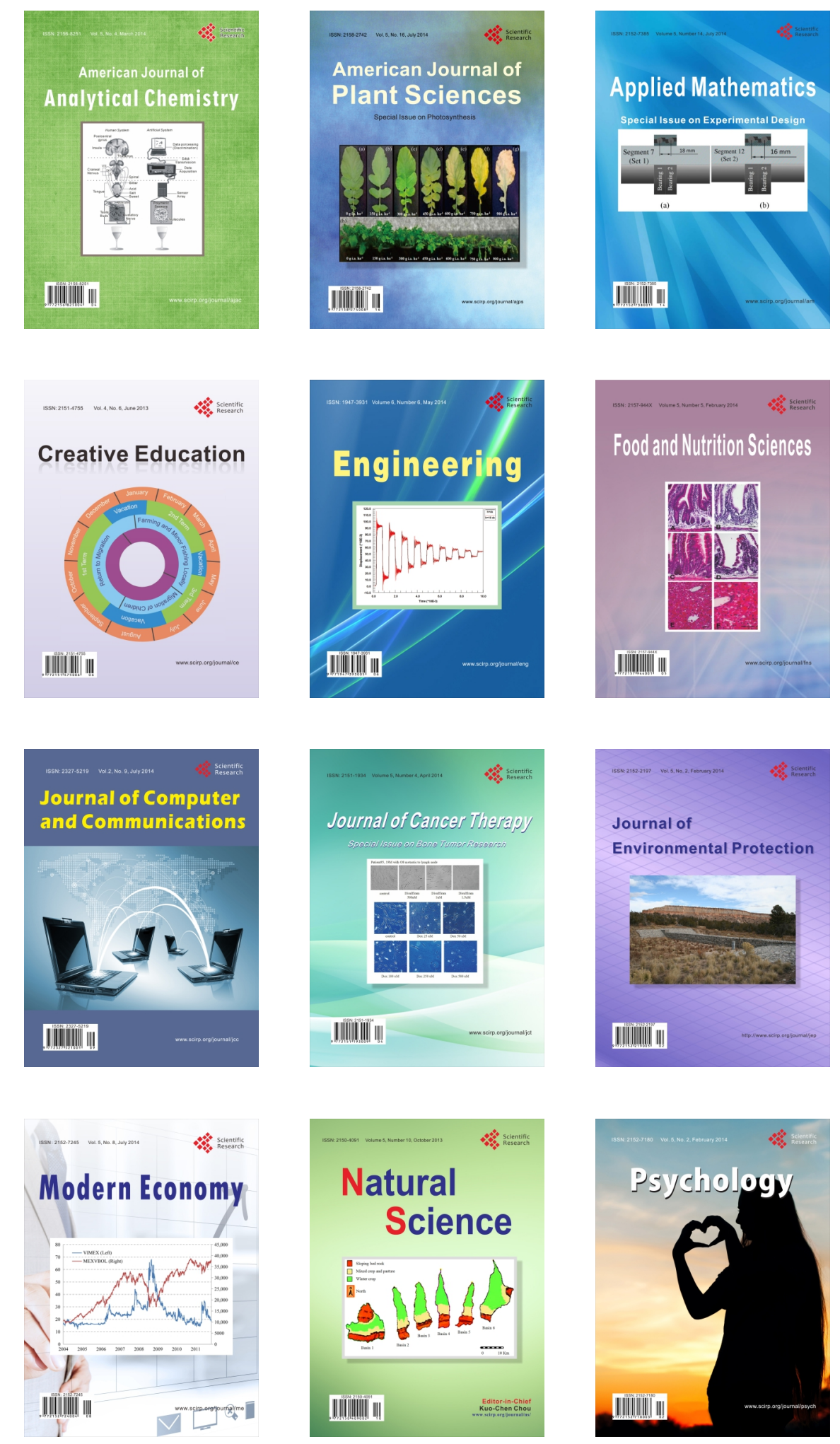